\title{
Understanding martensite and twin formation in austenitic steels: a model describing TRIP and TWIP effects
}

\author{
E.I. Galindo-Nava*, P.E.J. Rivera-Díaz-del-Castillo \\ Department of Materials Science and Metallurgy, University of Cambridge \\ 27 Charles Babbage Rd, Cambridge, CB3 0FS, UK \\ *email: eg375@cam.ac.uk, +44 1223334300
}

\begin{abstract}
A unified description for the evolution of $\epsilon^{-}$and $\alpha^{\prime}$ - martensite, and twinning in austenitic steels is presented. The formation of micron-scale $\epsilon$ and twin bands is obtained by considering the evolution of hierarchically arranged nano-sized $\epsilon$ and twins (embryos). The critical size and applied stress when these structures form is obtained by minimising their free energy of formation. The difference between forming an $\epsilon$ plate or a twin lies in the number of overlapping stacking faults in their structure. A nucleation rate criterion is proposed in terms of the critical embryo size, resolved shear stress and embryo number density. Based on Olson and Cohen's classical $\alpha^{\prime}$-martensite transformation model, the nucleation rate of $\alpha^{\prime}$ is considered proportional to that for $\epsilon$. These results, combined with dislocation-based approximations, are employed to prescribe the microstructure and flow stress response in steels where transformation-induced-plasticity (TRIP) and/or twinning-inducedplasticity (TWIP) effects operate; these include austenitic stainless and high-Mn steels. Maps showing the operation range of $\epsilon, \alpha^{\prime}$ and twinning in terms of the stacking fault energy at different strain levels are defined. Effects of chemical composition in the microstructure and mechanical response in stainless steels are also explored. These results allow identifying potential compositional scenarios when the TRIP and/or TWIP effects are promoted in austenitic steels.
\end{abstract}

Keywords: Austenite; Martensite; Deformation twinning; TRIP-assisted steel; Modelling

\section{Introduction}

Mechanical properties of austenitic steels depend strongly on the stability of the matrix. High strength, ductility and toughness can be achieved via the TRIP (transformationinduced plasticity) or TWIP (twinning-induced plasticity) effects where, respectively, 
strain-induced martensite and mechanical twinning form to accommodate additional strain. The occurrence of these mechanisms depends on the stacking fault energy $\left(\gamma_{S F E}\right)$, the initial microstructure and deformation conditions [1,2]; martensitic transformation from austenite $(\gamma)$ to $\epsilon-$ martensite and/or $\alpha^{\prime}$-martensite occurs if $\gamma_{S F E}$ is typically below $20 \mathrm{~mJ} \mathrm{~m} \mathrm{~m}^{-2}$, whereas mechanical twinning is promoted if the stacking fault energy lies between 15 and $30 \mathrm{~mJ} \mathrm{~m}^{-2}$. It has been reported that these structures form simultaneously if $\gamma_{S F E}$ lies between 15 and $20 \mathrm{~mJ} \mathrm{~m} \mathrm{~m}^{-2}$. For instance, Shen et al. [3] reported that $\epsilon, \alpha^{\prime}$ and twinning operate in 304SS $\left(\gamma_{S F E}=18 \mathrm{~mJ} \mathrm{~m}^{-2}\right)$ at different strains; $\epsilon$ and twinning act as intermediate phases in the transformation from $\gamma$ to $\alpha^{\prime}$-martensite. Analogous results have been obtained by other authors in steels with similar $\gamma_{S F E}$ values $[4-6]$. The reason for their simultaneous operation is that the mechanisms of formation and evolution of $\epsilon$ and twins are in fact similar [7]. $\epsilon$-martensite forms by the arrangement of intrinsic stacking faults on every second $\{111\}$ plane, whereas twins form by overlapping three stacking faults on successive planes [8]; stacking faults typically form by the dissociation of $\frac{1}{2}\langle 110\rangle\{111\}$ dislocations into $\frac{1}{6}\langle 112\rangle\{111\}$ Shockley partials. However, other authors have reported in C-containing TWIP steels possible twin formation by the formation of Frank partials [9]. The structure of $\epsilon$-martensite is different from the HCP phase in Fe forming at high pressures [10]; in the former, the structure consists of closely spaced stacking faults (deformation bands) within the matrix, whereas in the latter less microstructural defects have been observed, exhibiting a nearly uniform hexagonal structure. $\alpha^{\prime}$-martensite forms at the intersections of deformation bands $\left(\epsilon^{-}\right.$ martensite or twins) and its growth is confined to the extent of the bands [11]. Talonen and Hänninen [12] showed that $\alpha^{\prime}$ formation is also strongly influenced by the stacking fault energy.

Several models describing the evolution of $\epsilon / \alpha^{\prime}$ martensite [13-15] and twinning [16] and their effect on mechanical properties have been proposed. These formulations are based on considering a single mechanism (TRIP or TWIP) and there are few physics-based approximations considering both effects simultaneously as a function of the stacking fault energy. Dini et al. [17] used artificial neural networks to predict the mechanical response 
of Fe-Mn-Si-Al when TRIP and TWIP effects are present, however no microstructural information was included. Latypov et al. [18] have combined the finite-element method with phenomenological descriptions for dislocation, twinning and martensite evolution in a multiphase steel; although they were able to describe the strain hardening response and relative strain partitioning, no information on the effects of stacking fault energy in twinning or $\alpha^{\prime}$ formation were considered. This shows that, although these formulations are able to reproduce the behaviour of the evolving microstructures and mechanical properties, their application is valid only for specific alloying conditions (e.g. stacking fault energy). One of the reasons for this is the lack of a single framework being able to predict the occurrence and evolution of $\epsilon, \alpha^{\prime}$ and twinning, hence dictating the transitions where these structures operate.

The objective of this work is to introduce a unified description of $\epsilon$ - and $\alpha^{\prime}$ - martensite, and twinning in austenitic steels. It is based on postulating a mechanism for the formation of (micro) $\epsilon$ and twin bands being composed by hierarchical sequences of nano-sized $\epsilon$ and twins (embryos); the free energies for $\epsilon$ and twin formation are expressed in terms of the embryo width, stacking fault energy and applied stress in Section 3; the only difference between these energies is the number of stacking faults required for their nucleation. This allows determining the critical width of a stacking fault acting as nuclei for $\epsilon$ and/or twins. A nucleation rate criterion is proposed in terms of the critical width, resolved shear stress and number density of the nano structures. Based on Olson and Cohen's classical transformation model [19], the nucleation rate of $\alpha^{\prime}$ is considered proportional to that for $\epsilon$. These results are combined with dislocation-based descriptions to describe microstructure and flow stress where both TRIP and TWIP effects operate (Section 4). The model results are applied to various stainless and high-Mn austenitic steels in Section 5, predicting successfully the relative contribution to work hardening when more than one mechanism operates. Maps showing the operation range of $\epsilon, \alpha^{\prime}$ and twinning in terms of the stacking fault energy at different strain levels are shown in Section 6. Effects of chemical composition in the microstructure and mechanical response are also explored in this section. Concluding remarks are highlighted in Section 7. 


\section{Materials}

A number of stainless and Mn containing austenitic steels have been studied in this work. Table 1 shows their chemical composition and commercial denomination. Experimental results on the microstructure and mechanical response were obtained from the literature. For the case of 301SS and 304SS, a number of alloys were explored with different chemical compositions; specific compositions for each case will be provided throughout the manuscript.

\section{Theory}

\section{$3.1 \epsilon$ martensite and twin formation}

$\epsilon-$ martensite and twins form to accommodate excess strain from external loading. Previous experimental and theoretical studies indicate that an embryo of $\epsilon$ forms by an intrinsic stacking fault on every second $\{111\}$ planes $[11,20]$, whereas a twin embryo forms by overlapping three stacking faults on successive planes [8]. Although the interactions of $\epsilon$ and twins with the matrix and other crystal defects may differ, experimental evidence shows that their mechanisms of formation and evolution are in fact similar [7]. Fujita and Ueda [20] studied the mechanism of $\epsilon$ formation in Fe-18Cr-9.5Ni-1.5Mn-0.65Si-0.06C (wt \%) finding that $\epsilon$ plates grow by overlapping stacking faults on $\{111\}$ planes on every two atomic layers; they also found that once a stacking fault forms, adjacent faults can be easily activated promoting the growth of $\epsilon$ bands. More recently, Martin et al. [21] explored in detail the structure of $\epsilon$-martensite in a stainless steel with composition Fe15Cr-6Mn-6Ni-1Si-0.01C (wt\%) and stacking fault energy equal to $17.5 \mathrm{~mJ} \mathrm{~m} \mathrm{~m}^{-2}$. Using high-resolution TEM, they observed that deformation bands ( $\epsilon+$ twins) of several hundred nanometres were composed by periodic arrangements of stacking faults with less than $10 \mathrm{~nm}$ in thickness. They also observed a continuum transition between microstructural features where no sharp interfaces formed between $\epsilon$ and $\gamma$. Other authors have found similar arrangements of periodic nano-sized structures within $\epsilon$ bands [3]. Wei et al. [22] observed similar structures in a TWIP steel with composition Fe-28.64Mn-0.6C (wt\%); the stacking fault of this steel is in the range $20-30 \mathrm{~mJ} \mathrm{~m}^{-2}$. They reported that a 
micron-scale twin is composed by a hierarchical structure of overlapping nano-sized (6-8 nm) twins of constant width and clean boundaries, i.e. dislocation-free. Additionally, they found that not only the density of twins increases with strain but also their average width; the latter increases by overlapping additional nano-twins. Similar hierarchical structures of nano-twins have been observed by other authors [23].

These results indicate that the structures of micro- $\epsilon$ and twin bands consist of hierarchically arranged periodic sequences of nano-sized structures (embryos) and the bands grow by overlapping additional embryos. Following Fujita and Ueda's [20] analysis on $\epsilon^{-}$ martensite formation, the following mechanism is proposed for both $\epsilon$ and twin formation: i) an isolated stacking fault of width $r_{i}$ forms on a $\{111\}$ plane from the dissociation of a perfect dislocation under an applied stress (and positive strain rate $\dot{\varepsilon}>0$ ), as schematically illustrated in Figure 1(a). ii) The size of the stacking fault increases with applied stress until reaching a critical width $r_{i}^{*}$ (Figure $1(\mathrm{~b})$ ); the thickness $t_{i}$ of a stacking fault is typically $100 \mathrm{~nm}[3,12]$. iii) A number of stacking faults $n_{i}\left(n_{\epsilon}=1[24]\right.$ and $\left.n_{t}=3[8]\right)$ overlap in adjacent $\{111\}$ planes to form an embryo with a critical width $r_{i}^{*}$ and length $l_{i}^{*}$; this process aids in reducing the excess stress concentration from dislocation pile-ups [22]; this arrangement is schematically shown in Figure 1(b) for the case of an $\epsilon$ embryo and in (c) for a twin. $i v$ ) The length of the embryo $l_{i}$ increases and propagates through the grain interiors by subsequent overlapping of stacking faults in adjacent planes (Figure 1(d)).v) Once the structure has fully formed, its width $w_{i}$ increases by forming adjacent embryos of constant width $r_{i}^{*}$, as shown in Figure $1(\mathrm{e})$; this gives $w_{i}=r_{i}^{*} \hat{N}_{i}$, where $\hat{N}_{i}$ is the number of embryos in a band. vi) The increase in $\epsilon /$ twinning volume fraction increases by the formation and overlapping of new embryos in various locations of a grain leading to the formation of micro-bands (Figure 1(f)). The critical width to form a nano-sized embryo $r_{i}^{*}$ and the nucleation rate are required to describe microstructure evolution. The former can be estimated when a critical value for the energy of formation is reached and it depends on the stacking fault energy [19].

Kibey et al. [8] showed that the free energy of a twin embryo is composed by $i$ ) the strain energy of overlapping partials forming the nucleus, $\left.E_{d i s} ; i i\right)$ the energy associated 
with the nucleation of an embryo, $E_{\text {emb }}$; and $\left.i i i\right)$ the work done by the applied stress $\tau$ aiding in forming the stacking faults: $E_{\tau}=\frac{1}{m_{S F}} \tau b r_{i}^{2} n_{i}$ [8], where $r_{i}$ is the width of a stacking fault (forming embryo), $n_{i}$ is the number of stacking faults in an embryo, $b=0.25 \mathrm{~nm}$ is the magnitude of the Burgers vector and $m_{S F}$ is the Schmid factor of a partial forming in the $\langle 112\rangle\{111\}$ system. It is assumed that this analysis applies also to $\epsilon$ formation. The energy associated with stacking faults overlapping can be approximated by the energy of a pile-up [8]: $E_{d i s}=\frac{n_{i}^{2} \mu b^{2}}{8 \pi(1-\nu)} r_{i} \ln \left(\frac{r_{i}}{2 n_{i} l_{i}}\right)$, where $\mu=70$ GPa is the shear modulus and $\nu=0.31$ is Poisson's ratio; in this case $l_{i}^{*}=n_{i} b$ when $r_{i}=r_{i}^{*}$ (Figures 1(b) and (c)). Olson and Cohen [19] proposed that the energy for stacking fault nucleation (per unit area) is the difference between the stacking fault energy in the faulted planes $\left(\gamma_{S F E} n_{i}\right)$ and the interfacial energy between the FCC and HCP structure on each stacking fault $2 \sigma_{H C P} n_{i}[12,19]$; the energy of formation of an embryo is then $E_{e m b}=\left(\gamma_{S F E}-2 \sigma_{H C P}\right) r_{i} t_{i} n_{i}^{\dagger}$. This equation indicates that if the stacking fault energy is greater than the surface energy, the formation energy for $\epsilon$-martensite is unfavourable and additional dislocation overlapping on successive $\{111\}$ planes towards forming a twin is preferred. It is worth noting that an area $r_{i}^{2}$ is considered in $E_{\tau}$ as the applied stress promotes dislocation dissociation towards forming a stacking fault, whereas the area $r_{i} t_{i}$ is considered in $E_{e m b}$ as it describes the energy required to form an embryo from an arrangement of existing stacking faults. The free energy is expressed as:

$$
\begin{aligned}
E_{i} & =E_{d i s}+E_{e m b}-E_{\tau} \\
& =\frac{n_{i}^{2} \mu b^{2}}{8 \pi(1-\nu)} r_{i} \ln \left(\frac{r_{i}}{2 n_{i} l_{i}}\right)+\left(\gamma_{S F E}-2 \sigma_{H C P}\right) r_{i} t_{i} n_{i}-\frac{\tau b r_{i}^{2} n_{i}}{m_{S F}},
\end{aligned}
$$

where $i$ stands for $\epsilon$ or $\mathrm{t}$ (twin). For the calculations performed in this work, it will be assumed that the loading orientation is [111]; this gives $m_{S F}=0.31$ [25]. It is interesting noting that if the loading orientation is [001], the Schmid factor is 0.47 , and equation 1 shows that the stress required to reach similar $E_{i}$ values (than for [111]) is higher for this orientation. This is consistent with experimental evidence showing much lower twinning

\footnotetext{
${ }^{\dagger}$ Kibey et al. defined this energy based on the generalised planar fault energy obtained from ab initio simulations. The thermodynamic description of this term is analogous, as it contains the planar energies necessary for stacking fault nucleation.
} 
activity in 316SS under the [001] loading orientation compared to the [111] orientation [25]; similar conclusions have been obtained in Fe-22Mn-0.6C (wt\%) [26]. These results show that lower hardening response is expected under [001] orientation as the TRIP/TWIP effects are not favoured.

An embryo forms when a critical width $\left(r_{i}^{*}\right)$ is reached and the energy $E_{i}$ is minimised when:

$$
\frac{\partial E_{i}}{\partial r_{i}}=0
$$

Using the expressions given in equation 2, it leads to $\frac{\partial E_{d i s}}{\partial r_{i}}=\frac{n_{i}^{2} \mu b^{2}}{8 \pi(1-\nu)}\left(\ln \left(\frac{r_{i}}{2 n_{i} l_{i}}\right)+1\right)$, $\frac{\partial E_{e m b}}{\partial r_{i}}=\left(\gamma_{S F E}-\sigma_{H C P}\right) r_{i} t_{i} n_{i}$ and $\frac{\partial E_{\tau}}{\partial r_{i}}=\frac{2 \tau b r_{i}}{m_{S F}} n_{i}$. Combining these results with equation 2 , it gives a relationship between $r_{i}^{*}, n_{i}^{*}$ and $\tau$ to be:

$$
\frac{n_{i} \mu b^{2}}{8 \pi(1-\nu)}\left(\ln \left(\frac{r_{i}^{*}}{2 b n_{i}^{*}}\right)+1\right)+\left(\gamma_{S F E}-\sigma_{H C P}\right) r_{i}^{*} t_{i}-\frac{2 \tau b r_{i}^{*}}{m_{S F}}=0
$$

This equation can be solved numerically to obtain $r_{i}^{*}$ in terms of $\tau$ for a given stacking fault energy. As equation 3 has multiple solutions when $\tau$ increases, it is important identifying the critical condition when $E_{i}$ is minimum; $\epsilon$ nucleation is explored initially.

Figure 2(a) shows the possible solutions using equation 3 for $\epsilon\left(n_{\epsilon}=1\right)$ in a steel with stacking fault energy of $17.5 \mathrm{~mJ} \mathrm{~m} \mathrm{~m}^{-2}$ (black solid line); experimental evidence shows that the fraction of $\epsilon-$ martensite is higher than the fraction of twins for this condition $[3,12]$. The surface energy in austenitic steels has been estimated to be in the range 10-15 mJ $\mathrm{m}^{-2}[27,28] ; \sigma_{H C P}=11.5 \mathrm{~mJ} \mathrm{~m}^{-2}$ was adjusted for all calculations employed in this work. Experimental estimations of the width of an $\epsilon$ embryo are also shown for Fe-15Cr-6Mn6Ni-1Si-0.01C (wt\%) and 304SS obtained from [21] and [3], respectively; the former alloy has the same $\gamma_{S F E}$, whereas $\gamma_{S F E}$ in the latter ranges between 17 and $18 \mathrm{~mJ} \mathrm{~m}^{-2}$ [12]. The results show that the critical width increases with stress upon reaching a critical value of $r_{\epsilon}^{*}=4.7 \mathrm{~nm}$ when $\tau_{\epsilon}^{*}=36 \mathrm{MPa}$; lower stress levels are needed at higher widths. Moreover, $r_{\epsilon}^{*}$ corresponds to the size range of the nano- $\epsilon$ structures obtained from experiments. In addition, Staudhammer et al. [29] estimated an $\alpha^{\prime}$ embryo width of 5-7 nm in 304SS, being the model results consistent with their findings. To further support these results, 
Figure 2(b) shows the values of the free energy per unit area $\left(E_{\epsilon}\right.$ is divided by the area $\left.t_{\epsilon} b\right)$ at different stress levels. When $\tau=0 \mathrm{MPa}$ (black solid line), $E_{\epsilon}$ decreases with low $r_{\epsilon}$, however it increases rapidly as the embryo size increases becoming unstable $\left(E_{\epsilon}>0\right)$. When $\tau=\tau_{\epsilon}^{*}=36 \mathrm{MPa}$ (blue dashed line), the energy reaches a minimum at $r_{\epsilon}^{*}$ and it decreases thereafter, indicating that $\epsilon$ growth is stable. When $\tau=50 \mathrm{MPa}>\tau_{\epsilon}^{*}$ (red dotted line), no clear minimum is observed although the energy decreases with embryo size; this suggests that forming a new embryo should be more favourable than increasing its width when the applied stress increases; this is consistent with the proposed mechanism of $\epsilon$ formation and growth. It is interesting noting that if $\tau_{\epsilon}^{*}<\tau_{C R S S}$, where $\tau_{C R S S}$ is the critical resolved shear stress (Section 4), stacking faults form during early stages of plastic deformation; this is consistent with Talonen and Hänninen observations in 301LN showing deformation bands forming at low strains [12]. These results indicate that the critical embryo width corresponds to $r_{\epsilon}^{*}$ when the applied stress is $\tau_{\epsilon}^{*}$. In order to compare this result with the critical conditions for twin formation, the solution of the free energy of a twin embryo is also shown in Figure 2(a) (blue dashed line); the critical stress to form a twin is $51 \mathrm{MPa}$, showing that a higher stress is required for twin formation when $\gamma_{S F E}=17.5 \mathrm{~mJ} \mathrm{~m}^{-2}$ and $\epsilon$ nucleation is more prolific. It is interesting noting that although the experimental measurements of the stacking fault energy can differ slightly, the model is able to capture well the prevailing mechanisms. For instance, if $\gamma_{S F E}=17$ $\mathrm{mJ} \mathrm{m} \mathrm{m}^{-2}$, the critical radius and stress to form an $\epsilon$ embryo are $5.3 \mathrm{~nm}$ and $29 \mathrm{MPa}$, respectively, and when $\gamma_{S F E}=18 \mathrm{~mJ} \mathrm{~m}^{-2}$, these $r_{\epsilon}^{*}$ and $\tau_{\epsilon}^{*}$ are $3.5 \mathrm{~nm}$ and $43 \mathrm{MPa}$, respectively. Similarly for a twin embryo, if $\gamma_{S F E}=17 \mathrm{~mJ} \mathrm{~m}^{-2}$ the critical radius and stress are $10.8 \mathrm{~nm}$ and $47 \mathrm{MPa}$, respectively, whereas when $\gamma_{S F E}=18 \mathrm{~mJ} \mathrm{~m}^{-2}, r_{t}^{*}$ and $\tau_{t}^{*}$ equal $8.6 \mathrm{~nm}$ and $55 \mathrm{MPa}$, respectively. In both cases $r_{\epsilon}^{*} \geq r_{t}^{*}$ and $\tau_{\epsilon}^{*} \leq \tau_{t}^{*}$ indicating that $\epsilon$ formation is more prolific for this range of $\gamma_{S F E}$.

The same analysis is followed to study the critical conditions for twin formation when increasing the stacking fault energy; in this case $n_{t}=3$ in equations 1 and 3 , and a stacking fault energy of $21 \mathrm{~mJ} \mathrm{~m}^{-2}$ is considered. Figure 2(c) shows the solution of equation 3 with different stress values (blue solid line). Experimental estimations on the thickness of 
a nano twin are also shown for Fe-24Mn-0.6C with $\gamma_{S F E}$ to be within this range [22]. It is also observed that the critical width $r_{t}^{*}=6 \mathrm{~nm}$ at the maximum stress $\left(\tau_{t}^{*}=86 \mathrm{MPa}\right)$ is very close to the values obtained experimentally. Moreover, similar trends are obtained when plotting the variations of the free energy per unit area $\left(E_{t} /\left(t_{t} b\right)\right)$ with stacking fault width in Figure 2(d): it is only at $r_{t}^{*}$ when $\tau=\tau_{t}^{*}$, that the free energy reaches a minimum value (dashed line). This also confirms that $r_{t}^{*}$ should be the critical width of a nano-twin forming once the applied stress is $\tau_{t}^{*}$. In order to compare the conditions between twin and $\epsilon$ formation for this $\gamma_{S F E}$, the free energy of an $\epsilon$ embryo is also shown in Figure 2(c) (black dotted line); it is observed that much higher stress levels are required before an $\epsilon$ embryo can form (143 MPa), indicating that twin formation is more favourable in this case.

These results show that the width of nano-scaled $\epsilon$ and twins can be prescribed using the same energy balance and their occurrence differs by the number of stacking faults required to form each structure. Equation 3 shows that the applied stress to form an embryo increases with increasing the stacking fault energy. This indicates that for steels with low stacking fault energy lower applied stress levels are required to form a stacking fault; hence a lower dislocation density is needed, increasing the probability of forming an isolated stacking fault ( $\epsilon$ embryo). Conversely, if the stacking fault energy increases, higher dislocation density is promoted with increasing applied stress; this results in a higher probability for stacking fault/dislocation interactions promoting the formation of nanotwins. The relative contribution and competition between each mechanism are explored in detail in Section 6. It is worth mentioning that other dislocation dissociation reactions have been reported to influence twinning in TWIP steels containing high carbon additions [9]; this is due to the increase in local stress concentrations promoted by interstitial carbon atoms $[25,30]$. This is not considered in the present model to simplify the analysis, as a link between local stress concentration and carbon content would be needed in equation 1. Possible extensions to the model to include this are explored in the Discussion section. The results obtained in this section will allow describing the conditions for $\alpha^{\prime}$ martensite formation in terms of $\epsilon$ bands. 


\section{$3.2 \alpha^{\prime}$ martensite formation}

It is well established that $\alpha^{\prime}$-martensite nucleates at the intersections of deformation bands in metastable austenitic steels. The growth of $\alpha^{\prime}$ plates occurs by the repeated nucleation and coalescence of new $\alpha^{\prime}$ embryos [31]; this mechanism is analogous to that proposed for $\epsilon$ in the previous Section. Deformation bands can be composed by $\epsilon$-martensite and twins. It has been argued that $\alpha^{\prime}$ forms only in steels with stacking fault energy below $\sim 20 \mathrm{~mJ} \mathrm{~m}^{-2}[2]$, however van Tol et al. [32] have observed small fractions of $\alpha^{\prime}(\leq 1.2 \%)$ in a TWIP steel during deep drawing; the stacking fault energy calculated for this steel was $50 \mathrm{~mJ} \mathrm{~m} \mathrm{~m}^{-2}$. As the fraction of $\alpha^{\prime}$ is very low for this steel with high-stacking fault energy, it will be assumed that $\epsilon$ is the main precursor for the $\gamma \rightarrow \alpha^{\prime}$ transformation under uniaxial loading [3]. This is also supported by experiments showing that $\alpha^{\prime}$ and $\epsilon$ have the same dependence in the stacking fault energy [12]: as $\gamma_{S F E}$ increases, the overlapping of stacking faults becomes more irregular, and the nucleation of the $\alpha^{\prime}$ martensite becomes more difficult. Moreover, it was shown in the previous section that the width of an $\epsilon$ embryo is in the same width range than $\alpha^{\prime}$ embryos estimated experimentally [29]. Thus, it can be assumed that $\alpha^{\prime}$ forms from $\epsilon$ embryos and that the nucleation rate of $\alpha^{\prime}$ is linked to $\epsilon$ formation; this gives the size of an $\alpha^{\prime}$ embryo to be $r_{\alpha^{\prime}}^{*}=r_{\epsilon}^{*}$ and $t_{\alpha^{\prime}}=t_{\epsilon}=100$ nm when $\tau_{\alpha^{\prime}}^{*}=\tau_{\epsilon}^{*}$.

In Olson and Cohen's model for $\alpha^{\prime}$ transformation [11] it was assumed in the nucleation

rate of $\alpha^{\prime}$ embryos is proportional to the nucleation rate of $\epsilon$ bands, $\frac{d N_{\alpha^{\prime}}}{d t}=p \frac{d N_{\epsilon}}{d t}$, with $p$ being the probability of nucleating an embryo from the intersection. They assumed that $p$ was controlled by the temperature and stacking fault energy, however since these effects are captured via $r_{\alpha^{\prime}}^{*}$, it can be assumed that $p$ is constant. As two intersecting $\epsilon$ bands are required to form one $\alpha^{\prime}$ band, as a first approximation, the nucleation rate of $\alpha^{\prime}$ is proposed to evolve at half the rate of $\epsilon$, leading to:

$$
\frac{d N_{\alpha^{\prime}}}{d t}=\frac{1}{2} \frac{d N_{\epsilon}}{d t}
$$

This result will enable prescribing $\epsilon$ and $\alpha^{\prime}$ within the same formalism. Complete mi- 
crostructure evolution is presented in the following section.

\subsection{Microstructure evolution}

The critical size to form an embryo of $\epsilon, \alpha^{\prime}$ and twin were obtained in terms of the stacking fault energy and applied stress. In order to prescribe the evolution of these features, the nucleation rate $\frac{d N_{i}}{d t}$ and size of each structure are required. In the latter, $r_{i}^{*}$ and $t_{i}$ are constant and only their mean length is needed.

An evolution equation for the mean twin length $\left(l_{i}\right)$ with strain has been obtained in hexagonal closed-packed metals [33]. This was done by estimating the variation of $l_{i}$ with strain at the grain level, showing that it depends only on the nucleation rate of new embryos, as illustrated in Figure 1(d); the equation equals:

$$
\frac{d l_{i}}{d \varepsilon}=\frac{1}{N_{i}} \frac{d N_{i}}{d \varepsilon}\left(D-l_{i}\right)
$$

where $D$ is the average grain size, $N_{i}$ is the number density of nano-structures and $\frac{d N_{i}}{d \varepsilon}$ is the nucleation rate variation with axial strain; this equation is considered to be valid also for $\epsilon$ and $\alpha^{\prime}$, as it depends only on the nucleation rate for a given grain size.

In order to postulate expressions for the nucleation rate of an embryo, it is worth considering the processes affecting their formation, namely [33]: 1) Dislocation dissociation towards forming arrangements of stacking faults requires the formation and glide of $n_{i}$ perfect dislocations (Figure 1(a)); this process can be accounted for by considering the ratio $\frac{\tau_{n u c}}{n_{i} \tau_{C R S S}}$, where $\tau_{n u c}$ is the stress required to nucleate a dislocation (also referred to as friction stress) and $\tau_{C R S S}$ is the critical resolved shear stress to move that dislocation; the latter depends on composition, grain size and deformation conditions. 2) Increasing the width $\left(r_{i}^{*}\right)$ and number density $\left(N_{i}\right)$ of embryos will increase the frequency of dislocation pile-ups promoting the formation of new embryos [24]; this also promotes the formation of secondary deformation bands/twins on secondary slip planes [2]. 3) The number of nano-structures is limited by the volume fraction of the respective crystal defect. The nucleation rate for each structure is proposed in this work to scale with these factors in 
the phenomenological expression:

$$
\begin{aligned}
\frac{d N_{\epsilon}}{d t} & =\dot{\varepsilon} \frac{\tau_{n u c}}{\tau_{C R S S}} \frac{r_{\epsilon}^{*}}{b m_{S F}} N_{\epsilon}\left(1-f_{\epsilon}\right) \\
\frac{d N_{\alpha^{\prime}}}{d t} & =\frac{1}{2}\left(\dot{\varepsilon} \frac{\tau_{n u c}}{\tau_{C R S S}} \frac{r_{\alpha^{\prime}}^{*}}{b m_{S F}} N_{\alpha^{\prime}}\left(1-f_{\alpha^{\prime}}\right)\right) \\
\frac{d N_{t}}{d t} & =\dot{\varepsilon} \frac{\tau_{n u c}}{3 \tau_{C R S S}} \frac{r_{t}^{*}}{b m_{S F}} N_{t}\left(1-f_{t}\right) .
\end{aligned}
$$

Similar expressions have been derived to describe the twin nucleation rate in hexagonal closed packed metals with satisfactory agreement [33]. It is worth noting that $\frac{d N_{i}}{d \varepsilon}=\frac{1}{\dot{\varepsilon}} \frac{d N_{i}}{d t}$. Each equation is valid when the applied shear stress equals or exceeds $\tau_{i}^{*}$. Since $\tau_{C R S S}$ depends on the grain size, composition and deformation conditions (Section 4), equations 6 indicate that martensite and twinning is less likely to occur in fine-grained alloys, as higher stress levels are required for dislocations to propagate and promote stacking fault formation; this is consistent with experimental evidence showing that twin and $\alpha^{\prime}$ activity decreases with decreasing the grain size $[34,35]$. These features, as well as $\tau_{n u c}$ and $\tau_{C R S S}$ values are explored in the following section. The number of embryos in a deformation band $\hat{N}_{i}$ defined in Section 3.1 is linked to the number density through the relation $\hat{N}_{i}=\frac{1}{N_{i, 0}} N_{i}$ [36], where $N_{i, 0}$ is the number of nucleation sites. $N_{i, 0}$ in classical nucleation theory is assumed to be inversely proportional to the total volume of every single nucleation site [37], which in this case it would be the band limited by $r_{i}^{*} t_{i} D$, as $l_{i}$ increases up to $D$ (Figure $1(\mathrm{f})$ ); it will be assumed that this relation also holds for the (deformation-controlled) nucleation rate, giving $N_{i, 0}=\frac{0.05}{r_{i}^{*} t_{i} D}$, where the 0.05 factor was fitted to adjust all the experimental results tested in this work.

The volume fraction of each structure equals the product of its number density and the volume of an embryo $N_{i} V_{i}$; in addition, the term $\left(1-f_{\alpha^{\prime}}\right)$ is added to $f_{\epsilon}$ and $f_{t}$ to account for their reduction when $\alpha^{\prime}$ increases [3]. This results in the following relations:

$$
\begin{aligned}
f_{\epsilon} & =N_{\epsilon} V_{\epsilon}\left(1-f_{\alpha^{\prime}}\right)=N_{\epsilon} r_{\epsilon}^{*} t_{\epsilon} l_{\epsilon}\left(1-f_{\alpha^{\prime}}\right) \\
f_{\alpha^{\prime}} & =N_{\alpha^{\prime}} V_{\alpha^{\prime}}=N_{\alpha^{\prime}} r_{\alpha^{\prime}}^{*} t_{\alpha^{\prime}} l_{\alpha^{\prime}} \\
f_{t} & =N_{t} V_{t}\left(1-f_{\alpha^{\prime}}\right)=N_{t} r_{t}^{*} t_{t} l_{t}\left(1-f_{\alpha^{\prime}}\right)
\end{aligned}
$$


Solving equations 6 and 7 allows describing the structure of $\epsilon, \alpha^{\prime}$ and twinning with the same formalism.

\section{Dislocation evolution and mechanical response}

Prescribing dislocation evolution is necessary to quantify the flow stress response with strain; dislocation activity in the $\gamma$ is affected when the fraction of $\epsilon$ and $\alpha^{\prime}$ increases by accommodating additional strain. The variation in the total plastic strain $(d \varepsilon)$ can be described using a mixture rule between $\gamma$ and $\alpha^{\prime}[38]: d \varepsilon=d \varepsilon_{\gamma}\left(1-f_{\alpha^{\prime}}\right)+\varepsilon_{\alpha^{\prime}} d f_{\alpha^{\prime}} . \epsilon$ influences the strain accommodation by modifying $d \varepsilon_{\gamma}$, as it forms by the continuous stacking of dislocation partials. Additionally, since the austenite strength is usually much lower than the martensite, it can also be assumed that there is no plastic deformation in $\alpha^{\prime}$, i.e. $\varepsilon_{\alpha^{\prime}}=0$ [38], giving the relation $\frac{d \varepsilon_{\gamma}}{d \varepsilon}=\frac{1}{\left(1-f_{\alpha^{\prime}}\right)}$; this expression shows that strain accommodation in the matrix decreases as the fraction and $\alpha^{\prime}$ increase promoting the TRIP effect. The strain in the austenite is accommodated by dislocations, $\epsilon$ and deformation twins. An $\epsilon$ embryo forms by the stacking of single $\frac{1}{6}\langle 112\rangle$ partials dissociated from $\frac{1}{2}\langle 110\rangle\{111\}$ perfect dislocations and it can be considered that the strain accommodated by $\epsilon\left(d \varepsilon_{\epsilon}\right)$ is a fraction of the strain accommodated by dislocations $d \varepsilon_{d i s}$; since the strain energy of a Shockley partial is $\frac{2}{3}$ the energy of a perfect dislocation, this suggests that the strain accommodated by $\epsilon$ ammounts to a similar proportion [39], i.e. $d \varepsilon_{\epsilon}=\frac{2}{3} d \varepsilon_{d i s}$ is adopted. This gives the relation [16]: $d \varepsilon_{\gamma}=d \varepsilon_{d i s}\left(1-f_{t}-f_{\epsilon}\right)+\frac{1}{\sqrt{2} M} d f_{t}+\frac{2}{3} f_{\epsilon} d \varepsilon_{d i s}$, where $\frac{1}{\sqrt{2} M}$ term is the strain accommodated by a twin, and $M=3$ is the Taylor orientation factor [40]; rearranging the previous expression it gives $\frac{d \varepsilon_{d i s}}{d \varepsilon_{\gamma}}=\frac{1}{\left(1-f_{t}-\frac{1}{3} f_{\epsilon}\right)}\left(1-\frac{1}{\sqrt{2} M} \frac{d f_{t}}{d \varepsilon_{\gamma}}\right)$; this shows that dislocation contribution to strain accommodation decreases as the volume fraction of twins and $\epsilon$ increase, promoting the TWIP and TRIP effects, respectively. The evolution of $\rho$ can be prescribed in terms of the total strain $\varepsilon$ by combining the previous expressions [16]:

$$
\begin{aligned}
\frac{d \rho}{d \varepsilon} & =\frac{d \rho}{d \varepsilon_{d i s}} \frac{d \varepsilon_{d i s}}{d \varepsilon_{\gamma}} \frac{d \varepsilon_{\gamma}}{d \varepsilon}=\frac{d \rho}{d \varepsilon_{d i s}} \frac{1-\frac{1}{\sqrt{2} M} \frac{d f_{t}}{d \varepsilon_{\gamma}}}{\left(1-f_{t}-\frac{1}{3} f_{\epsilon}\right)} \frac{1}{\left(1-f_{\alpha^{\prime}}\right)} \\
& =\frac{d \rho}{d \varepsilon_{d i s}} \frac{1-\frac{1}{\sqrt{2} M} \frac{d f_{t}}{d \varepsilon}\left(1-f_{\alpha^{\prime}}\right)}{\left(1-f_{t}-\frac{1}{3} f_{\epsilon}\right)\left(1-f_{\alpha^{\prime}}\right)}
\end{aligned}
$$


$\frac{d \rho}{d \varepsilon_{d i s}}$ results from the competition between dislocation storage $\frac{d \rho+}{d \varepsilon_{d i s}}$ and recovery $\frac{d \rho-}{d \varepsilon_{d i s}}$. The latter is given by $f_{D R V} \rho$, where $f_{D R V}=3.5$ is the dynamic recovery coefficient and this value was adjusted to reproduce the experimental data explored in this work; similar values in austenitic steels have been reported by other authors [16].

When a number of impenetrable obstacles are present, the dislocation generation rate equals $[40,41]$ :

$$
\frac{d \rho+}{d \varepsilon_{d i s}}=\frac{k_{1}}{b} \sqrt{\rho}+\sum_{i} \frac{1}{\Lambda_{i} b} m_{S F},
$$

where the first term accounts for dislocation self-interactions, $\Lambda_{i}$ is the mean free path of each obstacle ( $\epsilon$ and twins) and $m_{S F}$ is the Schmid factor of $\langle 112\rangle\{111\}$ stacking faults forming from $\langle 110\rangle\{111\}$ perfect dislocations [25]; $k_{1}=0.06$ is a constant valid for austenitic steels $[40,42]$. For the case of hardening induced by deformation bands $(\epsilon$ or twinning), previous studies have considered that $\Lambda_{i}$ equals $[16,38]$ :

$$
\Lambda_{i}=\frac{2 w_{i}\left(1-f_{i}\right)}{f_{i}},
$$

where $w_{i}=r_{i}^{*} \hat{N}_{i}$ is the width of the respective crystal defect. Two assumptions have been introduced in the derivation of equations 9 and 10: 1) the twins or $\epsilon$ are fully formed along the grain interiors and 2) no effect of $\alpha^{\prime}$ forming within the bands is included. However, the length and distribution of the twins and $\epsilon$ bands increases with increasing their effective volume $N_{i} V_{i}=\frac{f_{i}}{1-f_{\alpha^{\prime}}}$ (equation 7 ), and the first assumption is removed via introducing this prefactor into equation 9. Additionally, since $\alpha^{\prime}$ grows within the bands, it can be considered that the effective band width $w_{i}$ decreases according to $1-f_{\alpha^{\prime}}$, i.e. the factor $\left(1-f_{\alpha^{\prime}}\right)$ is added to $\Lambda_{i}$ in equation 9 in order to account for $\alpha^{\prime}$ formation (assumption 2). The evolution of the dislocation density with strain accommodated by dislocations is given by:

$$
\frac{d \rho}{d \varepsilon_{d i s}}=\frac{k_{1}}{b} \sqrt{\rho}+\frac{m_{S F} f_{t}}{\Lambda_{t} b\left(1-f_{\alpha^{\prime}}\right)^{2}}+\frac{m_{S F} f_{\epsilon}}{\Lambda_{\epsilon} b\left(1-f_{\alpha^{\prime}}\right)^{2}}-f_{D R V} \rho
$$

This equation shows that the TRIP effect in dislocation hardening is directly influenced by 
the $\epsilon$ structure $\left(\Lambda_{\epsilon}\right)$; $\alpha^{\prime}$ affects the hardening response by replacing the deformation bands and by accommodating additional strain (equation 8). Microstructure evolution and work hardening response can be obtained by solving equations $6,7,8$ and 11 simultaneously. The flow stress can be estimated using Taylor's formula:

$$
\sigma=\sigma_{Y}+0.3 M \mu b \sqrt{\rho}
$$

where $\sigma_{Y}=\tau_{C R S S} M$ is the yield stress. This equation is employed to obtain the critical strain (at $\tau_{i}^{*}=\frac{1}{M} \sigma_{i}^{*}$ ) when $\epsilon, \alpha^{\prime}$ and/or twinning operate; the microstructural feature $i$ operates initially if $\tau_{i}^{*}<\tau_{C R S S}$.

$\sigma_{Y}$ in polycrystalline austenitic steels is dictated by the friction stress $\left(M \tau_{n u c}\right)$, solid solution hardening $\left(\sigma_{s s}\right)$ and Hall-Petch effects:

$$
\sigma_{Y}=\left(M \tau_{n u c}+\sigma_{s s}+\frac{k_{Y}}{\sqrt{D}}\right) G(T, \dot{\varepsilon})
$$

where $G$ is a function of the temperature and strain rate accounting for thermally activated effects altering dislocation glide [40]. A number of models based on thermal activation have been proposed to describe $T$ and $\dot{\varepsilon}$ effects in $\sigma_{Y}$ [43]. As this work is focused on describing microstructure evolution considering the yield stress as input, an empirical formula based on Kocks and Mecking model is adopted for the predictions [40]: $G(T, \dot{\varepsilon})=$ $10.3\left(\ln \frac{10^{7}}{\dot{\varepsilon}}\right)^{-0.7}\left(1.5-3.3 \times 10^{-4} T-8.9 \times 10^{-6} T^{2}+10^{-8} T^{3}\right)$, with $G \approx 1$ at room temperature $\left(20{ }^{\circ} \mathrm{C}\right.$ ) and $\dot{\varepsilon}=10^{-4} \mathrm{~s}^{-1}$ (quasi-static deformation). Solid solution hardening from substitutional elements is very low in austenitic steels $[4,44,45]$ and can be considered negligible; however $\mathrm{N}$ and $\mathrm{C}$ show significant hardening effects in $\sigma_{Y}[46,47]$; Fleischer's theory is employed to estimate the increment in the critical resolved-shear stress due to the presence of solute atoms $[48,49]: \sigma_{s s}=\left(\beta_{C}^{2} x_{C}+\beta_{N}^{2} x_{N}\right)^{1 / 2}$, where $x_{C}$ and $x_{N}$ are the atom fraction of $\mathrm{C}$ and $\mathrm{N}$ in the steel, respectively, and $\beta_{i}$ is the strengthening constant. $\beta_{i}$ values have been obtained from experiments: $\beta_{N}=1500 \mathrm{MPa} /$ at $[46]$ and $\beta_{C}=1000$ $\mathrm{MPa} /$ at [50]. The Hall-Petch constant in austenitic steels (stainless and Mn-containing) has been estimated to lie in the range $450-600 \mathrm{MPa} \mu \mathrm{m}^{1 / 2}$ when the grain size is above 
$\sim 2 \mu \mathrm{m}[50-52] ; k_{Y}=500 \mathrm{MPa} \mu \mathrm{m}^{1 / 2}$ is considered for the present calculations. $\tau_{n u c}$ in 316SS has been estimated to lie in the range $70-90 \mathrm{MPa}[25,53]$; however, the solid solution hardening contribution was not subtracted; it is estimated that the solid solution stress in this is steel is $\approx 60 \mathrm{MPa}$ and $\tau_{n u c}=15 \mathrm{MPa}$ is considered for the calculations.

Figure 3 shows a comparison between the predicted and measured yield stress for the alloys considered in Table 1; these conditions cover a wide range in chemical composition and grain sizes to test their contributions to strengthening. Additional experiments for $\sigma_{Y}$ at different temperatures, strain rates, and $\mathrm{C}$ and $\mathrm{N}$ additions are also included; measurements for Fe-20Mn-3Al-3Si (wt \%) at different temperatures and strain rates were obtained from [4], where $T$ ranges between $-76{ }^{\circ} \mathrm{C}$ and $400{ }^{\circ} \mathrm{C}$ and $\dot{\varepsilon}$ between $10^{-4}$ $\mathrm{s}^{-1}$ and $1000 \mathrm{~s}^{-1}$. $\sigma_{Y}$ values for the family FeCrNiMn-(0.24-0.56)N of austenitic steels containing N up to 0.56 wt\% were obtained from [47]. Results for the Fe-Mn-Al-C family containing different $\mathrm{Mn}, \mathrm{Al}$ and $\mathrm{C}$ additions are displayed as Fe-(12-30)Mn(0-1.4)Al-(01.2)C; these include experimental values for the alloys shown in Table 1 and additional results in Fe-Mn-C alloys with $\mathrm{C}$ content up to 1.2 wt\% (obtained from [50]); no grain boundary strengthening is considered in the latter, as coarse grains were reported. The model shows very good correlation in the trends with a maximum discrepancy of $\sim 75$ $\mathrm{MPa}$ in the alloy Fe-20Mn-1.2C (wt\%). The correlation coefficient is 0.94, indicating that equation 10 reproduces well the experiments. This enables exploring the role of deformation conditions and grain size in the microstructure evolution, via equation 6 .

\section{Results}

The model results for $\epsilon, \alpha^{\prime}$ and twinning are tested against experiments for a number of steels (Table 1); different deformation conditions are considered for each material. The stacking fault energy is a critical input parameter for the models and these values are taken from experiments; $\gamma_{S F E}$ values at room temperature $\left(20^{\circ} \mathrm{C}\right)$ were obtained from the literature and are shown in Table 2. For the case of Fe16Cr9Ni6Mn1Si, Ulrich et al. [54] assumed $\gamma_{S F E}=26 \mathrm{~mJ} \mathrm{~m}^{-2}$, however this steel forms $\epsilon$ and $\alpha^{\prime}$, indicating that the stacking fault energy should be lower. Moreover, Weidner et al. [55] have measured 
$\gamma_{S F E}=17.5 \mathrm{~mJ} \mathrm{~m}^{-2}$ in a steel with similar composition (Fe-16Cr-6Mn-6Ni in wt \%); based on experimental and theoretical studies on alloying effects in $\gamma_{S F E}[56,57]$, the additions of 1 wt\% Si and $3 \mathrm{Ni}$ wt\% in Fe16Cr9Ni6Mn1Si (compared to Fe-16Cr-6Mn$6 \mathrm{Ni}$ ) should increase the stacking fault energy by $\approx 1.5 \mathrm{~mJ} \mathrm{~m}^{-2}$, leading to $\gamma_{S F E}=19$ $\mathrm{mJ} \mathrm{m} \mathrm{m}^{-2}$; details of this estimation are shown in Section 6.1. $\gamma_{S F E}$ shown for 204SS was estimated in [58] using thermodynamic principles. For the case of Fe20Mn3Si3Al no experimental estimations were found. Pierce et al. [28] have measured $\gamma_{S F E}$ in steels with similar composition (Mn in the range 20-24 wt\% with different Si and Al content), being in the range of $12-18 \mathrm{~mJ} \mathrm{~m}^{-2} ; \gamma_{S F E}=17 \mathrm{~mJ} \mathrm{~m}^{-2}$ was adopted for Fe20Mn3Si3Al. The model results will also be tested at different temperatures and this effect has to be introduced. Remy et al. [59] have determined from experiments in stainless steels that $\gamma_{S F E}$ has linear variation with temperature in the range 100-600 K; the slope range is $0.04-0.08 \mathrm{~mJ} \mathrm{~m}^{-2} / \mathrm{K}$. Miodownik et al. [60] found similar slope values in stainless steels in the range 200-400 K. Based on these results, the following relation for the stacking fault energy at different temperatures is adopted for austenitic stainless steels $\gamma_{S F E}=\gamma_{S F E}^{R T}+0.05(T-293)$. MATLAB scripts with the solution of all models are included as supplementary material.

The model results for twinning are explored first. Figure 4 shows the predicted and measured twinned structure at room temperature in Fe22Mn0.6C, including (a) average twin width, (b) twin spacing, (c) volume fraction and (d) mechanical response; equation 10 was used in (b). The deformation conditions for this steel are $\dot{\varepsilon}=5 \times 10^{-4} \mathrm{~s}^{-1}$ and $D=50 \mu \mathrm{m}$; the average twin width is estimated using the relation $\sqrt{\frac{w_{t} t_{t}}{2}}$ to account for stereological corrections when measuring the mean width [61]. The model shows very good agreement in (a), (b) and (c), confirming that the microstructure is well described at different length scales, including the nano- and micron-scale twin width and average spacing. The predicted twin width $\left(14-30 \mathrm{~nm}\right.$ when $\left.f_{t}=0-5 \%\right)$ is also in agreement with experimental results in Fe-24Mn-0.6C (wt\%) obtained in [22]. The flow stress is underpredicted by $\sim 150 \mathrm{MPa}$ at $\varepsilon=50 \%$, and this discrepancy can be due to $\mathrm{C}$ additions promoting additional strain hardening and other dislocation dissociations, as 
previously reported by other authors $[30,50,62]$; additional results in steels with low $\mathrm{C}$ will be shown in Figure 7 to support that TWIP effects can be described.

Figure 5 shows the model predictions in 301LN and 304LN, where $\alpha^{\prime}$ operates. Figures 5(a) and (b) show the volume fraction evolution and flow stress response, respectively, in $301 \mathrm{LN}$ at room temperature and different strain rates. The composition of this alloy is Fe-17.6Cr-6.6Ni-1.61Mn-0.48Si-0.22Cu-0.14Mo-0.019C (wt \%) and $D=11 \mu \mathrm{m}[12]$. The $\alpha^{\prime}$ volume fraction at $3 \times 10^{-4} \mathrm{~s}^{-1}$ increases up to $95 \%$ at $\varepsilon=35 \%$, whereas the predicted maximum twin volume fraction is $15 \%$ and the maximum fraction of $\epsilon$ is $60 \%$. Similar values for twinning and $\epsilon$ are predicted at other strain rates. These result indicate that the flow stress response in this steel is controlled by strain-induced $\alpha^{\prime}$ and $\epsilon$ formation (TRIP effect). The model shows very good agreement for $f_{\alpha^{\prime}}$, confirming that the $\alpha^{\prime}$ nucleation rate is sensitive to the strain rate (via $\left.\tau_{C R S S}\right)$. The model predicts higher flow stress at higher strains, nevertheless it successfully captures negative strain rate sensitivity observed. The latter can be due to a competition between dislocation forest and stacking fault development; at low strain rates the dislocation generation rate is lower, hence promoting the probability of dislocation dissociation and $\epsilon$ formation, whereas at high strain rates a high dislocation interaction rate could prevent forming stacking faults. The discrepancies in the model can be due to the assumption of the constant probability of an $\alpha^{\prime}$ plate to nucleate at the intersection of two $\epsilon$ bands not being held at higher strains.

Additional results for $f_{\alpha^{\prime}}$ in 304LN with composition Fe-18.2Cr-8.1Ni-1.71Mn-0.33Si$0.37 \mathrm{Cu}-0.32 \mathrm{Mo}-0.04 \mathrm{C}(\mathrm{wt} \%)$ are shown in Figure $5(\mathrm{c}) ; D=5 \mu \mathrm{m}$ is reported in this case. The model predicts higher $\alpha^{\prime}$ fraction by up to $10 \%$; nevertheless, it successfully captures $f_{\alpha^{\prime}}$ variation with strain rate; the discrepancies can be due to local variations in the grain orientation [25]. Figure 5(d) shows the $\alpha^{\prime}$ variation with temperature in $301 \mathrm{LN}$ at $\dot{\varepsilon}=3 \times 10^{-4} \mathrm{~s}^{-1}$. The temperature affects $\alpha^{\prime}$ formation via $\gamma_{S F E}$ and $\tau_{C R S S}$; between $-40{ }^{\circ} \mathrm{C}$ and $80{ }^{\circ} \mathrm{C}$ the former ranges from $11.5 \mathrm{~mJ} \mathrm{~m}^{-2}$ to $17.5 \mathrm{~mJ} \mathrm{~m} \mathrm{~m}^{-2}$, whereas the predicted values of $\tau_{C R S S}$ using equation $14\left(\sigma_{Y}=\tau_{C R S S} M\right)$ are $126 \mathrm{MPa}$ and 87 $\mathrm{MPa}$, respectively; no experimental values of $\sigma_{Y}$ were reported. The stacking fault energy controls mostly microstructure evolution, as $r_{\alpha^{\prime}}^{*}$ varies exponentially with $\gamma_{S F E}$ (equation 
3). The model successfully predicts $f_{\alpha^{\prime}}$ within these temperatures. These results show that the formulation correlates well $\alpha^{\prime}$ evolution with the flow stress response when the TRIP effect is the main hardening mechanism. Combining the results shown in Figures 4 and 5 will enable describing microstructure and mechanical response in steels where different mechanisms operate simultaneously.

Figures 6(a) and (b) show the predicted and measured volume fraction of $\epsilon, \alpha^{\prime}$ and twinning in 304SS and Fe16Cr9Mn6Ni1Si, respectively; the composition of 304SS is Fe14.35Cr-8.71Ni-1.93Mn-0.36Si-0.79Cu-0.027C (at\%) [3], the grain size is $25 \mu \mathrm{m}$ and the stacking fault energy is assumed equal to that for 304LN (Table 2); $D=20 \mu \mathrm{m}$ in Fe16Cr9Mn6Ni1Si. In both cases, the model reproduces the experimental measurements with good agreement; in (a) it is predicted that the fraction of $\epsilon$ and twinning increases with strain up to $\sim 35-40 \%$; above this strain their fraction decreases due to $\alpha^{\prime}$ formation. Shen et al. [3] observed in this steel that $\epsilon$ forms first at low strains $(\varepsilon \leq 20 \%)$, this is followed by twinning $(\varepsilon \leq 30 \%)$ and $\alpha^{\prime}$ dominates at strains above $\varepsilon=30 \%$. The predictions are in good agreement with their observations showing that the fraction of $\epsilon$ is greater for low strains and it is later consumed by the $\alpha^{\prime}$. It is worth noting that very low $\alpha^{\prime}$ fraction was reported and predicted in 304LN in Figure 5(c), whereas in this case the $\alpha^{\prime}$ fraction is significantly higher; the difference is due to the grain size being $5 \mu \mathrm{m}$ in 304LN reducing significantly the nucleation rate of $\epsilon$ and $\alpha^{\prime}$, whereas in 304SS $D=25 \mu \mathrm{m}$. In Figure 6(b), the model is able to reproduce the relative variation in $\epsilon$ and $\alpha^{\prime}$ fraction, confirming that the link between the nucleation rate of $\epsilon$ and $\alpha^{\prime}$ proposed in equation 4 is also in agreement with experiments. Although the twinning volume fraction was not reported in this steel, high twinning activity was observed during deformation [3]. Figure 6(c) shows the corresponding flow stress response in 304SS and Fe16Cr9Mn6Ni1Si with the microstructures shown in (a) and (b). The model shows good agreement in the flow stress variation and slope change of 304SS when $\epsilon, \alpha^{\prime}$ and twinning operate simultaneously, however lower flow stress is predicted in Fe16Cr9Mn6Ni1Si; the discrepancies can be due to the activation of secondary slip and twinning systems not considered in the model which can modify the hardening rate, as reported by Ulrich et al. [54]. Figure 6(d) shows 
additional results on the evolution of the twinning volume fraction in Fe20Mn1.2C and Fe17Mn0.56C1.4Al. In both cases the model predicts lower volume fractions, although the relative variation of $f_{t}$ with $\gamma_{S F E}$ is consistent. The discrepancies can be due to different dislocation reactions promoting twin formation with high $\mathrm{C}$ additions [9]. It has been pointed out that carbon promotes twin formation not only by increasing the stacking fault energy, but also by pinning the dislocation partials and increasing the local stress concentration; the latter aids in promoting the formation of Frank partials, instead of Schockley partials [25], hence modifying the conditions for twin formation and evolution (equations 1 and 6). This effect has not been included in the present model, nevertheless it could be considered in future work.

Another interesting aspect to explore with the model is the role of the grain size and stacking fault energy in microstructure evolution. Figure 7 shows a comparison between the model and experiments for (a) $f_{\alpha^{\prime}}$ and (b) the flow stress response in 204SS with different grain sizes. The model shows good agreement for low strain levels $(\varepsilon \leq$ $30 \%$ ), however higher $\alpha^{\prime}$ fraction was reported at higher strains. Additionally, the model underpredicts $\sigma_{Y}$ values by $70 \mathrm{MPa}$ and it estimates lower flow stress response in both cases, although the shape change in the steel with higher $\alpha^{\prime}$ volume fraction is well reproduced. The discrepancies can be due to the assumption of the probability of an $\alpha$ ' plate to nucleate at the intersection of two $\epsilon$ bands being constant, and the $\epsilon$ bands can promote additional strain hardening. Another possibility is due to an inhomogeneous grain or solute distribution, as these alloys were $\gamma$-reversion annealed from cold-rolled lath martensite (to obtain different grain sizes); Kisko et al. [34] observed that retained and reverted austenite were present in some testing conditions, altering locally the stability in the austenitic matrix. In order to explore the role of the stacking fault energy in the macroscopic stress response and compare the TRIP and TWIP effects, Figures 7(c) and (d) show the volume fraction and flow stress response in 3 FeMn3Si3Al steels with different Mn contents, respectively. It has been confirmed that TRIP operates in Fe20Mn3Si3Al, TWIP occurs in Fe25Mn3Si3Al, and only dislocation glide is active in Fe30Mn3Si3Al [4] (see Table 2); only the experimental volume fraction of $\alpha^{\prime}$ in Fe20Mn3Si3Al was reported 
in this case. $f_{t}$ in Fe25Mn3Si3Al is lower than $f_{\alpha^{\prime}}$ in Fe20Mn3Si3Al, hence inducing lower work hardening; this is confirmed in Figure $7(\mathrm{~d})$. The model reproduces well the relative variation in the flow stress when different mechanisms operate, however it underpredicts the dislocation hardening response in Fe30Mn3Si3Al. The higher measured yield stress and initial work hardening in Fe20Mn3Si3Al can be due to the presence of $\sim 20 \%$ of thermal $\epsilon$ martensite prior deformation, increasing the dislocation/stacking fault activity in the material. The predictions improve as the $\alpha^{\prime}$ fraction increases, as this initial effect decreases with $f_{\alpha^{\prime}}$.

\section{Discussion}

A unified description in the formation of $\epsilon, \alpha^{\prime}$ and mechanical twinning in austenitic steels has been presented. These structures were shown to be formed by hierarchical arrangements of nano-sized embryos. The critical size $\left(r_{i}^{*}\right)$ and applied stress $\left(\tau_{i}^{*}\right)$ for their formation were obtained by minimising their energy of formation. The only difference between forming an $\epsilon$ or twin embryo is in the number of overlapping stacking faults required to form a nucleus. The critical width of an $\alpha^{\prime}$ plate was considered to be the same as an $\epsilon$ embryo, as the latter controls $\alpha^{\prime}$ formation. The nucleation rate was proposed to be proportional to the critical width, critical resolved shear stress and number density of embryos. The nucleation rate of $\alpha^{\prime}$ was considered proportional to that for $\epsilon$, following Olson and Cohen's transformation model [19]. These results are consistent with Staudhammer et al. [29] observations, showing that the critical size of an $\alpha^{\prime}$ embryo did not depend on the deformation conditions and it is only by the applied stress that they form, i.e. the deformation conditions affect the nucleation rate only. This analysis is similar to classical nucleation theories based on thermal activation [36], however in this case both $r_{i}^{*}$ and $\frac{d N_{i}}{d t}$ are deformation-controlled. The macroscopic flow stress response was obtained using conventional dislocation theory where twins and/or $\epsilon$ bands act as impenetrable obstacles; $\alpha^{\prime}$ influenced the flow stress by affecting the strain accommodated in the $\gamma$ and by replacing the $\epsilon$ and twin bands as impenetrable obstacles for dislocation slip. Using this formulation it was possible to describe the microstructure and plastic response in 
austenitic stainless and high-Mn steels with different initial microstructures and stacking fault energies; good agreement was shown when TRIP and/or TWIP effects operate. The loading direction was considered parallel to the [111] orientation. Nevertheless, the model, in principle, is able to describe orientation effects in the evolution of the microstructure and stress-strain response. For instance, if the results shown in Figures 6(a) and (c) for 304SS are considered in the [001] loading orientation, lower $\alpha^{\prime}, \epsilon$ and twinning activity are predicted by the model. The calculated volume fractions at $\varepsilon=50 \%$ are $f_{\alpha^{\prime}}=26 \%$, $f_{\epsilon}=57 \%$ and $f_{t}=33 \%$, whereas the volume fractions shown in Figure 6(a) for the [111] orientation at this strain are $f_{\alpha^{\prime}}=55 \%, f_{\epsilon}=44 \%$ and $f_{t}=36 \%$. As for the hardening response, the predicted flow stresses at $\varepsilon=50 \%$ in the [111] and [001] orientation are $1690 \mathrm{MPa}$ and $1400 \mathrm{MPa}$, respectively, showing lower hardening on the [001] orientation. Although the model successfully predicts a decrease in the hardening response due to the lower $\alpha^{\prime}, \epsilon$ and twinning fraction in the [001] orientation, as experimentally observed by other authors [63], effects on dislocation evolution are not included in the model. Adopting additional modelling methods such as crystal plasticity or viscoplastic self-consistent approaches would enable including the effects of orientation, multiple slip and activation of secondary twinning on individual grains.

The model was able to predict partially the evolution of twinning in $\mathrm{C}$-containing TWIP steels. The discrepancies can be due to carbon affecting the dislocation dissociation reactions to form twins. For instance, Idrissi et al. [9] observed a high density of sessile dislocations (Frank partials) at the twin bands in a high-C TWIP steel (Fe-20Mn-1.2C wt\%), whereas in a C-free steel (Fe-28Mn-3.5Si-2.8Al wt\%) very few were observed. Twin nucleation by a Frank partial can occur if it is combined with a Shockley partial and a Cottrell-Lomer (CL) lock, however high local stresses are required for this process to occur [25,64-66]. Karaman et al. [25] proposed that this mechanism occurs in a Hadfield steel (Fe-12.34Mn-1C-0.9Si-0.38Cr-0.46Ni wt\%) due to the high carbon additions decrease the mobility of the lock and increase the local stress concentrations. Other authors have reached similar conclusions in steels with medium or high carbon content $[30,63]$. These features can affect the energy balance proposed in equation 1 by including a stress term 
promoted by carbon atoms and by modifying the interaction energy between dissociating partials $\left(E_{d i s}\right)$. This extension can be done in future work. Additionally, the evolution of the total twin volume fraction was considered in this work and possible effects of secondary twinning have not been included explicitly. Nevertheless, it has been shown in previous work that [33], in principle, the model can be extended to account for the individual evolution of secondary and primary twins and account for their respective effects in the work hardening response. This can also be done in future work by accounting for the relevant dislocation dissociation processes and reorientation effects of the primary twins [67].

A number of authors have defined maps showing the regimes where each mechanism operates in terms of the stacking fault energy [1,2]. Although, they provide qualitative insights for their range of operation, their evolution is also controlled by the applied strain $[3,54]$. Using this formulation it is possible to explore the effect of the stacking fault energy in the relative activity of $\epsilon, \alpha^{\prime}$ and twinning with different strain levels. Figure 8 shows the variation in volume fraction of these structures for different stacking fault energy values at (a) $\varepsilon=20 \%$ and (b) $\varepsilon=60 \%$; the conditions tested for this case are $\dot{\varepsilon}=10^{-3} \mathrm{~s}^{-1}, T=20{ }^{\circ} \mathrm{C}, D=20 \mu \mathrm{m}$, and no solid solution hardening is considered. The regions where each mechanism dominates, based on previous studies, are also highlighted in (b); the predicted map is in good agreement with these studies. The map differs between low and high strains for $\gamma_{S F E}$ in the range $16-22 \mathrm{~mJ} \mathrm{~m}^{-2}$, where transitions occur. For instance, when $\gamma_{S F E}=18 \mathrm{~mJ} \mathrm{~m}^{-2}$ the volume fraction of $\epsilon$ and twinning increase rapidly in (a) $(\sim 20 \%)$, however these structures transform into $\alpha^{\prime}$ as the strain increases in (b). Similarly, when $\gamma_{S F E}=20 \mathrm{~mJ} \mathrm{~m}^{-2}, f_{t}$ and $f_{\epsilon}$ have similar values in $(\mathrm{a})(\sim 12-15 \%)$, however the twin volume fraction increases rapidly up to $70 \%$ as the strain increases. These results are in agreement with the predicted microstructures for the steels explored in the previous section: $\alpha^{\prime}$ dominates in 301LN and 204SS due to their low $\gamma_{S F E}$ (Table 2), whereas $\epsilon, \alpha^{\prime}$ and twinning operate jointly in 304SS and Fe16Cr9Ni6Mn1Si as they have intermediate $\gamma_{S F E}$ values. Twinning is the dominating mechanism in Fe20Mn1.2C, Fe22Mn0.6C, Fe17Mn1.4Al0.6C and Fe25Mn3Si3Al, whereas 
dislocation glide is only present in Fe30Mn3Si3Al. It is also possible exploring how the chemical composition affects the occurrence of these mechanisms if the stacking fault energy is known.

\subsection{Alloying contributions to microstructure evolution}

The main contribution of alloying additions to microstructure evolution is through the stacking fault energy. $\gamma_{S F E}$ values were obtained from experimental measurements and remained fixed for a given composition. It is interesting exploring how the microstructure changes when modifying alloying additions from a base composition. Several studies on alloying effects in the stacking fault energy have been performed using various experimental techniques, thermodynamic principles, and atomistic simulations [56, 57,68-70]. Although, there is significant scatter between predicted and measured values for wide compositional ranges, linear relations between composition and $\gamma_{S F E}$ exist for small compositional variations. Thus, the stacking fault energy can be approximated by:

$$
\gamma_{S F E}=\gamma_{S F E}^{0}+\sum_{j} \alpha_{j}\left(x_{j}-x_{j}^{0}\right)
$$

where $\gamma_{S F E}^{0}$ and $x_{j}^{0}$ are the stacking fault energy and atom fraction of the alloying element $j$ in the base alloy and $x_{j}$ is the atom fraction of the variant composition. Effects of $\mathrm{Ni}$, Mn, Si and Cr in austenitic stainless steels are explored and Table 3 shows the coefficients employed in this work which have been obtained by other authors. $\alpha_{N i}$ in Table 3 is valid only when adding up to $12 \mathrm{wt} \%$, whereas $\alpha_{M n}$ is valid when $\mathrm{Ni}$ content is in the range 8-10 wt \%.

Figure 9(a) and (b) show the effect in the volume fraction and flow stress response, respectively, in 304SS with $3 \mathrm{Ni}$ additions (8.3, 9 and 12 wt\%). The stacking fault energy for the base composition is considered equal to $304 \mathrm{LN}$ and the predicted values for the remaining alloys are $19.4 \mathrm{~mJ} \mathrm{~m}^{-2}$ and $24.8 \mathrm{~mJ} \mathrm{~m}^{-2}$. The model shows good agreement in the variation of $f_{\alpha^{\prime}}$ and flow stress with stacking fault energy. Figures $9(\mathrm{c})$ and (d) show the variation in $\alpha^{\prime}$ volume fraction in 301SS at different temperatures and at $\varepsilon=30 \%$ when modifying (c) Si and Mn and (d) Ni and Cr simultaneously; the 
experimental results were obtained from [71]. In this case, the stacking fault energy for the base composition (Fe-17.34Cr-7.03Ni-1.07Mn-0.48Si-0.11C-0.02N wt\%) is assumed to be $14.7 \mathrm{~mJ} \mathrm{~m}^{-2}$ (Table 2); specific compositional variations for each condition can be found in [71]; the model shows good agreement in the trends for all temperatures in (c), however it predicts lower $f_{\alpha^{\prime}}$ values when increasing/decreasing $\mathrm{Ni} / \mathrm{Cr}$ content in (d); the discrepancies can be due to Cr affecting the contribution of $\mathrm{Ni}$ to the stacking fault energy [57]; this has not been included in equation 11. Nevertheless, the results show that the model is sensitive to compositional variations if they are captured properly in the stacking fault energy. These results also show that different mechanisms can be tailored when modifying alloying additions. For instance in 304SS (assuming base composition Fe-18Cr-8Ni0.33Si wt $\%$ and $\gamma_{S F E} \approx 17.8 \mathrm{~mJ} \mathrm{~m}^{-2}$ ), increasing Si or Cr to $0.96 \mathrm{wt} \%$ and $20.8 \mathrm{wt} \%$, respectively, promotes $\alpha^{\prime}$ formation and the TRIP effect $\left(\gamma_{S F E} \approx 15 \mathrm{~mJ} \mathrm{~m}{ }^{-2}\right)$, whereas increasing $\mathrm{Ni}$ up to $10.5 \mathrm{wt} \%$ reduces the formation of $\epsilon$ and $\alpha^{\prime}$, promoting the TWIP effect only $\left(\gamma_{S F E} \approx 22 \mathrm{~mJ} \mathrm{~m}^{-2}\right)$. This framework could be applied to alloy design for TRIP/TWIP effects if alloying effects are considered in $\gamma_{S F E}$.

\section{Conclusions}

- A unified description for the evolution of $\epsilon$ - and $\alpha^{\prime}$ - martensite, and twinning in austenitic steels was presented. These results, combined with dislocation-based descriptions, were applied to describe microstructure and flow stress response in steels where TRIP and/or TWIP operate. The description was able to capture the competition between different microstructural features in stainless and high-Mn steels.

- The evolution of micron-scale $\epsilon$ and twin bands were obtained by describing a hierarchical arrangement of nano-sized $\epsilon$ and twins (embryos). The critical size and applied stress when these structures form were obtained by minimising the free energy of formation. The only difference between forming an $\epsilon$ plate or a twin was in the number of overlapping stacking faults. The evolution of twinning in TWIP steels with medium/high-carbon was underpredicted; a reason for this can be due 
different dislocation reactions occurring by the presence of carbon atoms, altering the critical energy for twin nucleation. These features can be explored more in detail in the future.

- A nucleation rate equation was proposed in terms of the critical embryo size, resolved shear stress and number density. The nucleation rate of $\alpha^{\prime}$ was considered proportional to the rate of $\epsilon$ nucleation. This criterion was consistent with Olson and Cohen's transformation model [19].

- Mechanism maps showing the operation range of $\epsilon, \alpha^{\prime}$, and twinning in terms of the stacking fault energy were identified at different strain levels. Deformation temperature and chemical composition effects in microstructure were explored via modifying the stacking fault energy.

- The role of chemical composition in austenitic stainless steels was explored, showing that TRIP and TWIP effects can be promoted in the same alloying system. For instance in 304SS, increasing Si or Cr to $0.96 \mathrm{wt} \%$ or $20.8 \mathrm{wt} \%$, respectively, promotes $\alpha^{\prime}$ formation $\left(\gamma_{S F E} \approx 15 \mathrm{~mJ} \mathrm{~m}^{-2}\right)$, whereas increasing Ni up to $10.5 \mathrm{wt} \%$ promotes

TWIP effect only $\left(\gamma_{S F E} \approx 22 \mathrm{~mJ} \mathrm{~m}^{-2}\right)$. This shows that the present work can be applied to alloy design for TRIP/TWIP effects.

\section{Acknowledgements}

This research was supported by the grant EP/L025213/1 from the UK Engineering and Physical Sciences Research Council (EPSRC).

\section{References}

[1] H. Schumann, Neue Hütte 17 (1972) 605-609.

[2] S. Allain, J. Chateau, O. Bouaziz, S. Migot, N. Guelton, Correlations between the calculated stacking fault energy and the plasticity mechanisms in the $\mathrm{Fe}-\mathrm{Mn}-\mathrm{C}$ alloys, Mater Sci Eng A 387-389 (2004) 158-162.

[3] Y. Shen, X. Li, X. Sun, Y. Wang, L. Zuo, Twinning and martensite in a 304 austenitic stainless steel, Mater Sci Eng A 552 (2012) 514-522.

[4] O. Grässel, L. Krüger, G. Frommeyer, L. Meyer, High strength Fe-Mn-(Al,Si) TRIP/TWIP steels development - properties - application, Int J Plasticity 16 (2000) 1391-1409. 
[5] H. Ding, Z. Tang, W. Li, M. Wang, D. Song, Microstructures and mechanical properties of Fe-Mn-(Al,Si) TRIP/TWIP steels, J Iron Steel Res 13 (2006) 66-70.

[6] Y. Shen, N. Jia, Y. Wang, X. Sun, L. Zuo, D. Raabe, Suppression of twinning and phase transformation in an ultra fine grained $2 \mathrm{GPa}$ strong metastable austenitic steel: experiment and simulation, Acta Mater 97 (2015) 305-315.

[7] H. Idrissi, K. Renard, L. Ryelandt, D. Schryvers, P. Jacques, On the mechanism of twin formation in Fe-Mn-C TWIP steels, Acta Mater 58 (2010) 2464-2476.

[8] S. Kibey, J. Liu, D. Johnson, H. Sehitoglu, Predicting twinning stress in fcc metals: linking twin-energy pathways to twin nucleation, Acta Mater 55 (2007) 6843-6851.

[9] H. Idrissi, K. Renard, D. Schryvers, P. Jacques, On the relationship between the twin internal structure and the work-hardening rate of TWIP steels, Scripta Mater 63 (2010) 961-964.

[10] S. Ackermann, S. Martin, M. Schwarz, C. Schimpf, D. Kulawinski, C. Lathe, S. Henkel, D. Rafaja, H. Biermann, A. Weidner, Investigation of phase transformations in high-alloy austenitic TRIP steel under high pressure (up to $18 \mathrm{GPa}$ ) by In Situ synchrotron X-ray diffraction and Scanning Electron Microscopy, Metall Mater Trans A 47 (2016) 95-111.

[11] G. Olson, M. Cohen, Kinetics of strain-induced martensitic nucleation, Metall Trans A 6 (1975) 791-795.

[12] J. Talonen, H. Hänninen, Formation of shear bands and strain-induced martensite during plastic deformation of metastable austenitic stainless steel, Acta Mater 55 (2007) 6108-6118.

[13] C. Garion, B. Skoczeń, S. Sgobba, Constitutive modelling and identification of parameters of the plastic strain-induced martensitic transformation in 316L stainless steel at cryogenic temperatures, Int J Plas 22 (2006) 1234-1264.

[14] H. Han, C. Lee, D. Suh, S. Kim, A microstructure-based analysis for transformation induced plasticity and mechanically induced martensitic transformation, Mater Sci Eng A 485 (2008) 224-233.

[15] K. Lee, M. Chun, M. Kim, J. Lee, A new constitutive model of austenitic stainless steel for cryogenic applications, Comp Mater Sci 46 (2009) 1152-1162.

[16] O. Bouaziz, N. Guelton, Modelling of TWIP effect on work-hardening, Mater Sci Eng A 319-321 (2001) 246-249.

[17] G. Dini, A. Najafizadeh, S. Monir-Vaghefi, A. Ebnonnasir, Predicting of mechanical properties of Fe-Mn-(Al,Si) TRIP/TWIP steels using neural network modeling, Comp Mater Sci 45 (2009) 959-965.

[18] M. Latypov, S. Shin, B. De Cooman, H. Kim, Micromechanical finite element analysis of strain partitioning in multiphase medium manganese TWIP+TRIP steel, Acta Mater 108 (2016) 219-228. 
[19] G. Olson, M. Cohen, A general mechanism of martensitic nucleation: part I. General concepts and the FCC $\rightarrow$ HCP transformation, Metall Trans A 7 (1976) 1897-1904.

[20] H. Fujita, S. Ueda, Stacking faults and F.C.C. $(\gamma) \rightarrow$ H.C.P. $(\epsilon)$ transformation in 18/8-type stainless steel, Acta Metall 20 (1972) 759-767.

[21] S. Martin, C. Ullrich, D. Rafaja, Deformation of austenitic CrMnNi TRIP/TWIP steels: nature and role of the $\epsilon$-martensite, Mater today: proceedings $2 \mathrm{~S}$ (2015) 643-646.

[22] Y. Wei, Y. Li, L. Zhu, Y. Liu, X. Lei, G. wang, Y. Wu, Z. Mi, J. Liu, H. Wang, H. Gao, Evading the strength-ductility trade-off dilemma in steel through gradient hierarchical nanotwins, Nature communications (2014) 3580.

[23] P. Zhou, Z. Liang, R. Liu, M. Huang, Evolution of dislocations and twins in a strong and ductile nano twinned steel, Acta Mater 111 (2016) 96-107.

[24] J. Brooks, M. Loretto, R. Smallman, Direct observations of martensite nuclei in stainless steel, Acta Metall 27 (1979) 1839-1847.

[25] I. Karaman, H. Sehitoglu, H. Maier, Y. Chumnlyakov, Competing mechanisms and modeling of deformation in austenitic stainless steel single crystals with and without nitrogen, Acta Mater 49 (2001) 3919-3933.

[26] I. Gutierrez-Urrutia, S. Zaefferer, D. Raabe, The effect of grain size and grain orientation on deformation twinning in a Fe-22 wt.\% Mn-0.6 wt.\%C TWIP steel, Mater Sci Eng A 527 (2010) 3552-3560.

[27] D. Geissler, J. Freudenberger, S. Kauffmann, Martin, D. Rafaja, Assessment of the thermodynamic dimension of the stacking fault energy, Phil Mag 94 (2015) 29672979 .

[28] D. Pierce, J. Jiménez, J. Bentley, D. Raabe, C. Oskay, J. Witting, The influence of manganese content on the stacking fault and austenite/ $\epsilon$-martensite interfacial energies in Fe-Mn-(Al-Si) steels investigated by experiment and theory, Acta Mater 68 (2014) 238-253.

[29] K. Staudhammer, L. Murr, S. Hecker, Nucleation and evolution of strain-induced martensitic (b.c.c.) embryos and substructure in stainless steel: a transmission electron microscope study, Acta Metall 31 (1983) 267-274.

[30] M. Koyama, T. Sawaguchi, K. Tsuzaki, Deformation twinning behavior of twinninginduced plasticity steels with different carbon concentrations - part 2: proposal of dynamic-strain-aging-assisted deformation twinning, ISIJ int 55 (2015) 1754-1761.

[31] L. Murr, K. Staudhammer, S. Hecker, Effects of strain state and strain rate on deformation-induced transformation in 304 stainless steel: Part II. Microstructures study, Metall Trans A 13 (1982) 627-635.

[32] R. van Tol, J. Kim, L. Zhao, J. Sietsma, B. De Cooman, $\alpha^{\prime}$-Martensite formation in deep-drawn Mn-based TWIP steel, J Mater Sci 47 (2012) 4845-4850. 
[33] E. Galindo-Nava, Modelling twinning evolution during plastic deformation in hexagonal close-packed metals, Mater Design 83 (2015) 327-343.

[34] A. Kisko, R. Misra, J. Talonen, L. Karjalainen, The influence of grain size on the strain-induced martensite formation in tensile straining of an austenitic $15 \mathrm{Cr}-9 \mathrm{Mn}-$ Ni-Cu stainless steel, Mater Sci Eng A 578 (2013) 408-416.

[35] K. Rahman, V. Vorontsov, D. Dye, The effect of grain size on the twin initiation stress in a TWIP steel, Acta Mater 89 (2015) 247-257.

[36] D. Porter, K. Easterling, M. Sherif, Phase transformations in metals and alloys, CRC press, 2009.

[37] E. Kozeschnik, Modeling solid-state precipitation, Momentum press, 2013.

[38] A. Perlade, O. Bouaziz, Q. Furnémont, A physically based model for TRIP-aided carbon steels behaviour, Mater Sci Eng A 356 (2003) 145-152.

[39] D. Hull, D. Bacon, Introduction to dislocations, Butterworth-Heinemann, 1999.

[40] U. Kocks, H. Mecking, Physics and phenomenology of strain hardening: the FCC case, Prog Mater Sci 48 (2003) 171.

[41] Y. Estrin, H. Mecking, A unified phenomenological description of work hardening and creep bad on one-parameter models, Acta Metall 32 (184) 57-70.

[42] P. Rivera-Díaz-del-Castillo, K. Hayashi, E. Galindo-Nava, Computational design of nano structured steels employing irreversible thermodynamics, Mater Sci Tech 29 (2013) 1206.

[43] D. Caillard, J. Martin, Thermally activated mechanisms in crystal plasticity, Pergamon, 2003.

[44] K. Park, K. Jin, S. Han, S. Hwang, K. Choi, C. Lee, Stacking fault energy and plastic deformation of fully austenitic high manganese steels: effect of Al addition, Mater Sci Eng A 527 (2010) 3651-3661.

[45] D. Ryoo, N. Kang, C. Kang, Effect of Ni content on the tensile properties and straininduced martensite transformation for 304 stainless steel, Mater Sci Eng A 528 (2011) $2277-2281$.

[46] E. Kozlov, L. Teplyakova, N. Koneva, V. Gavrilyuk, N. Popova, L. Ignatenko, G. Fedoseeva, S. Smuk, A. Paul, V. Podkovka, Interactions in the dislocation ensemble in the formation of flow stress in austenitic nitrogen steel, Russian Phys J 39 (1996) 211-229.

[47] E. Werner, Solid solution and grain size hardening of nitrogen-alloyed austenitic steels, Mater Sci Eng A 101 (1988) 93-98.

[48] R. Fleischer, Substitutional solution hardening, Acta Metall 11 (1963) 203.

[49] R. Labusch, A statistical theory of solid solution hardening, Phys Stat Sol 41 (1970) 659-669. 
[50] O. Bouaziz, H. Zurob, B. Chehab, J. Embury, S. Allain, M. Huang, Effect of chemical composition on work hardening of Fe-Mn-C TWIP steels, Mater Sci Tech 27 (2011) 707-709.

[51] K. Singh, S. Sangal, G. Murty, Hall-Petch behaviour of 316L austenitic stainless steel at room temperature, Mater Sci Tech 18 (2002) 165-172.

[52] C. Sinclair, D. Marechal, J. Mithieux, The effect of grain size on the mechanical response of a metastable austenitic stainless steel, MATEC Web of Conf 7 (2013) 01010 .

[53] G. Monnet, M. Pouchon, Determination of the critical resolved shear stress and the friction stress in austenitic stainless steels by compression of pillars extracted from single grains, Mater Letters 98 (2013) 128-130.

[54] C. Ullrich, R. Eckner, L. Krüger, S. Martin, V. Klemm, D. Rafaja, Interplay of microstructure defects in austenitic steel with medium stacking fault energy, Mater Sci Eng A 649 (2016) 390-399.

[55] A. Weidner, C. Segel, H. Biermann, Magnitude of shear of deformation-induced $\alpha^{\prime}-$ martensite in high-alloy metastable steel, Mater Letters 143 (2015) 155-158.

[56] K. Jeong, J. Jin, Y. Jung, S. Kang, Y. Lee, The effects of Si on the mechanical twinning and strain hardening of Fe-18Mn-0.6C twinning-induced plasticity steel, Acta Mater 61 (2013) 3399-3410.

[57] L. Vitos, J. Nilsson, B. Johansson, Alloying effects on the stacking fault energy in austenitic stainless steels from first-principles theory, Acta Mater 54 (2006) 38213826 .

[58] S. Curtze, V. Kuokkala, A. Oikari, J. Talonen, N. Hänninen, Thermodynamic modellng of the stacking fault energy of austenitic steels, Acta Mater 59 (2011) 1068-1076.

[59] L. Remy, A. Pineau, Temperature dependence of stacking fault energy in close-packed metals and alloys, Mater Sci Eng 36 (1978) 47-63.

[60] A. Miodownik, The calculation of stacking fault energies in Fe-Ni-Cr alloys, CALPHAD 2 (1978) 207-226.

[61] L. Chang, H. Bhadeshia, Austenite films in bainitic microstructures, Mater Sci Tech 11 (1995) 874-881.

[62] M. Huang, O. Bouaziz, D. Barbier, S. Allain, Modelling the effect of carbon on deformation behaviour of twinning induced plasticity steels, J Mater Sci 46 (2011) $7410-7414$.

[63] I. Kireeva, Y. Chumlyakov, Orientation dependence of the $\gamma-\epsilon$ martensitic transformation in single crystals of austenitic stainless steels with low stacking fault energy, Phys Met Metallogr 191 (2006) 186-203.

[64] M. Azzaz, J. Michel, A. George, Plastic deformation, extended stacking faults and deformation twinning in single crystalline indium phosphide, Phil Mag A 69 (1994) 903-924. 
[65] J. Hirth, R. Hoagland, Extrinsically dissociated dislocations in simulated aluminium, Phil Mag A 78 (1998) 529-532.

[66] M. Baskes, R. Hoagland, T. Tsuji, An atomistic study of the strength of an extendeddislocation barrier, Modell Sim Mater Sci Eng 6 (1998) 9-18.

[67] Y. Cao, Y. Wang, Z. Chen, X. Liao, M. Kawasaki, S. Ringer, T. Langdon, Y. Zhu, De-twinning via secondary twinning in face-centered cubic alloys, Mater Sci Eng A 578 (2013) 110-114.

[68] R. Schramm, R. Reed, Stacking fault energies of seven commercial austenitic stainless steels, Metall Trans A 6 (1975) 1345-1351.

[69] J. Jin, Y. Lee, Effects of $\mathrm{Al}$ on microstructure and tensile properties of C-bearing high Mn TWIP steel, Acta Mater 60 (2012) 1680-1688.

[70] S. Lu, Q. Hu, B. Johansson, L. Vitos, Stacking fault energies of Mn, Co and Nb alloyed austenitic stainless steels, Acta Mater 59 (2011) 5728-5734.

[71] K. Nohara, Y. Ono, N. Ohashi, Composition and grain size dependencies of straininduced martensitic transformation in metastable austenitic stainless steel, ISIJ 63 (1972) 212-222.

[72] J. Talonen, P. Nenonen, G. Pape, H. Hänninen, Effect of strain rate on the straininduced $\gamma \rightarrow \alpha^{\prime}$-martensite transformation and mechanism properties of austenitic stainless steels, Metall Mater Trans A 36 (2005) 421-432.

[73] A. Soulami, K. Choi, Y. Shen, W. Liu, X. Sun, M. Khaleel, On deformation twinning in a $17.5 \%$ Mn-TWIP steel: a physically based phenomenological model, Mater Sci Eng A 528 (2011) 1402-1408.

[74] K. Renard, H. Idrissi, D. Schryvers, P. Jacques, On the stress state dependence of the twinning rate and work hardening in twinning-induced plasticity steels, Acta Mater 66 (2012) 966-971.

[75] I. Gutierrez-Urrutia, D. Raabe, Dislocation and twin substructure evolution during strain hardening of an Fe-22 wt.\% Mn-0.6 wt.\% C TWIP steel observed by electron channeling contrast imaging, Acta Mater 59 (2011) 6449-6442.

[76] S. Vercammen, Processing and tensile behaviour of TWIP steels, microstructural and textural analysis, Ph.D. thesis, Leuven: Katholieke Universiteit Leuven (2004).

[77] A. Das, Revisiting stacking fault energy of steels, Metall Mater Trans A 47 (2016) $748-768$. 
Table 1: Chemical composition (in wt\%) of the steels tested in this work.

\begin{tabular}{cccccccccc}
\hline Steel & $\mathrm{Ni}$ & $\mathrm{Mn}$ & $\mathrm{Si}$ & $\mathrm{Al}$ & $\mathrm{C}$ & $\mathrm{N}$ & $\mathrm{Cr}$ & $\mathrm{Cu}$ & Author \\
\hline 301SS & $6.6-9.2$ & $0.5-1.6$ & $0.15-0.88$ & - & 0.02 & $0.09-0.12$ & $15.9-18.7$ & 0.22 & {$[12,71,72]$} \\
304SS & $8-12$ & 1.7 & $0.33-0.72$ & - & $0.02-0.06$ & $0.05-0.1$ & 18.3 & $0.4-0.79$ & {$[3,6,12,45,72]$} \\
Fe16Cr9Ni6Mn1Si & 8.85 & 5.56 & 0.96 & - & 0.05 & 0.06 & 15.75 & - & {$[54]$} \\
204SS & 1.1 & 9 & 0.4 & - & 0.079 & 0.115 & 15.2 & 1.68 & {$[34]$} \\
Fe17Mn1.4Al0.6C & - & 17.5 & 0.24 & 1.4 & 0.56 & - & - & - & {$[73]$} \\
Fe20Mn1.2C & - & 20 & - & - & 1.2 & - & - & & {$[74]$} \\
Fe20Mn3Si3Al & - & 20 & 3 & 3 & - & - & - & & {$[4]$} \\
Fe22Mn0.6C & - & 22 & - & - & 0.6 & - & - & & {$[75]$} \\
Fe25Mn3Si3Al & - & 25 & 3 & 3 & - & - & - & & {$[4]$} \\
Fe30Mn3Si3Al & - & 30 & 3 & 3 & - & - & - & & {$[4]$} \\
\hline
\end{tabular}


Table 2: Values of the experimental stacking fault energy.

\begin{tabular}{ccc}
\hline Steel & $\gamma_{S F E} \mathrm{~mJ} \mathrm{~m}^{-2}$ & Author \\
\hline 301SS & 14.7 & {$[12]$} \\
304SS & 17.8 & {$[12]$} \\
204SS & 16.8 & {$[58]$} \\
Fe16Cr9Ni6Mn1Si & 19 & {$[54,55]$} \\
Fe20Mn1.2C & 20 & {$[74]$} \\
Fe17Mn1.4Al0.6C & 27 & {$[69]$} \\
Fe22Mn0.6C & 22 & {$[75]$} \\
Fe20Mn3Si3Al & 17 & {$[4]$} \\
Fe25Mn3Si3Al & 21 & {$[28]$} \\
Fe30Mn3Si3Al & 40 & {$[76]$} \\
\hline
\end{tabular}


Table 3: Stacking fault energy parameters

\begin{tabular}{ccc}
\hline Element & $\alpha_{j}\left(\mathrm{~mJ} \mathrm{~m}^{-2} / \mathrm{wt} \%\right)$ & Author \\
\hline $\mathrm{Ni}$ & 1.8 & {$[57,77]$} \\
$\mathrm{Cr}$ & -0.9 & {$[77]$} \\
$\mathrm{Mn}$ & -1.5 & {$[70]$} \\
$\mathrm{Si}$ & -4 & {$[69]$} \\
\hline
\end{tabular}


(a)

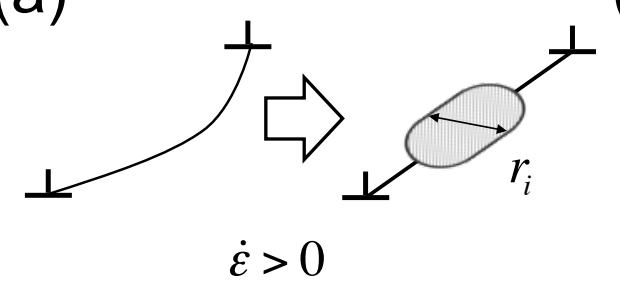

(b)

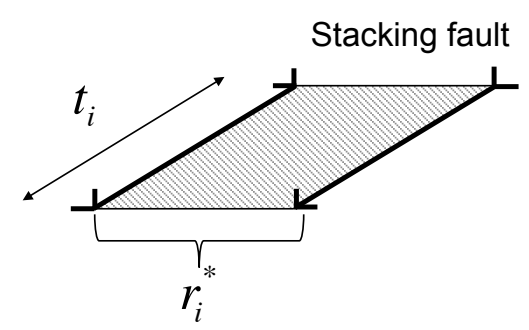

(c)

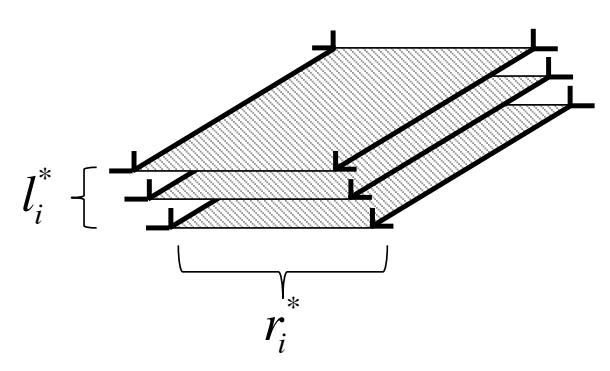

(d)

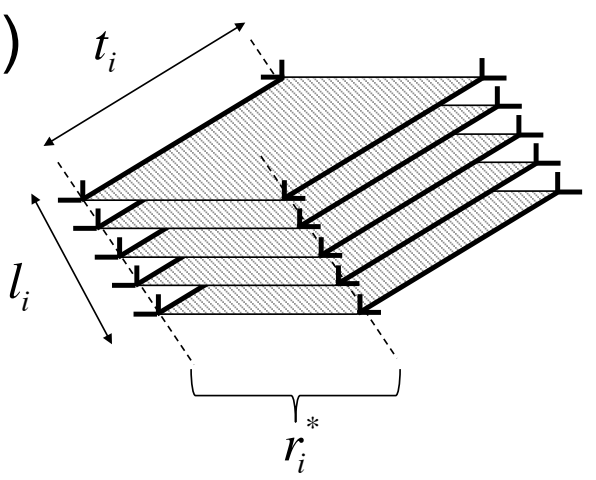

(e)

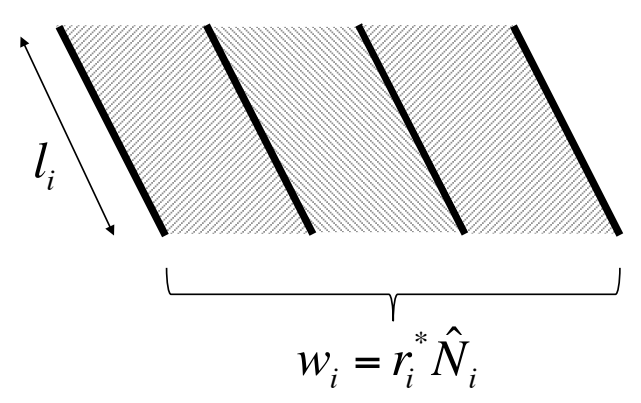

(f)

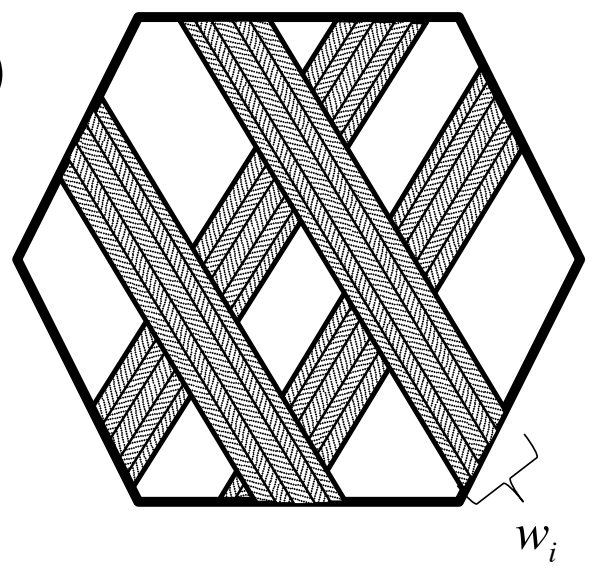

Figure 1: Schematic representation of the proposed mechanism of $\epsilon$ and twin formation. 

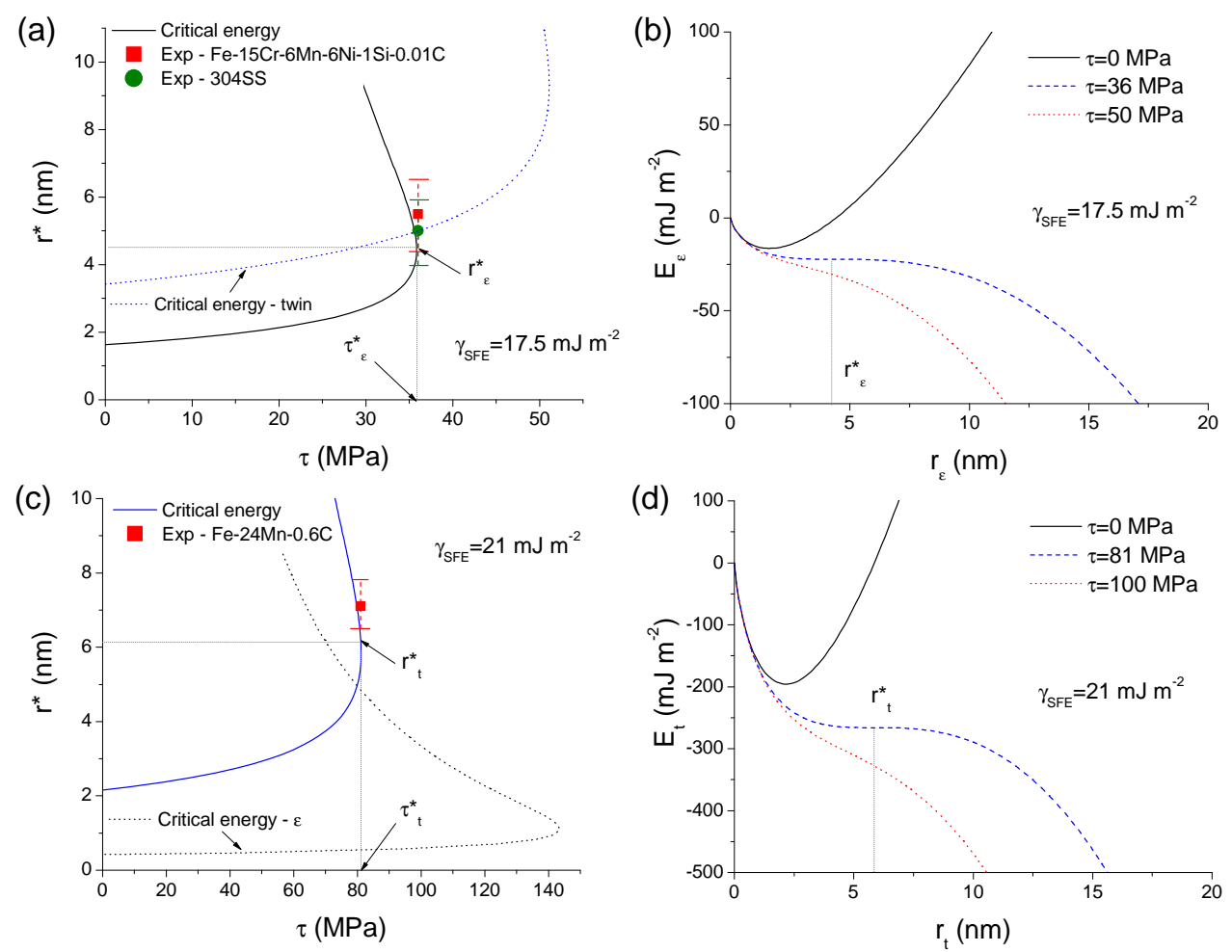

Figure 2: (a) Critical $\epsilon$ width vs applied stress. (b) Free energy variations with $\epsilon$ width for different stress levels. (c) Critical nano-twin width $v s$ applied stress. (d) Free energy variations with nano-twin width for different stress levels. 


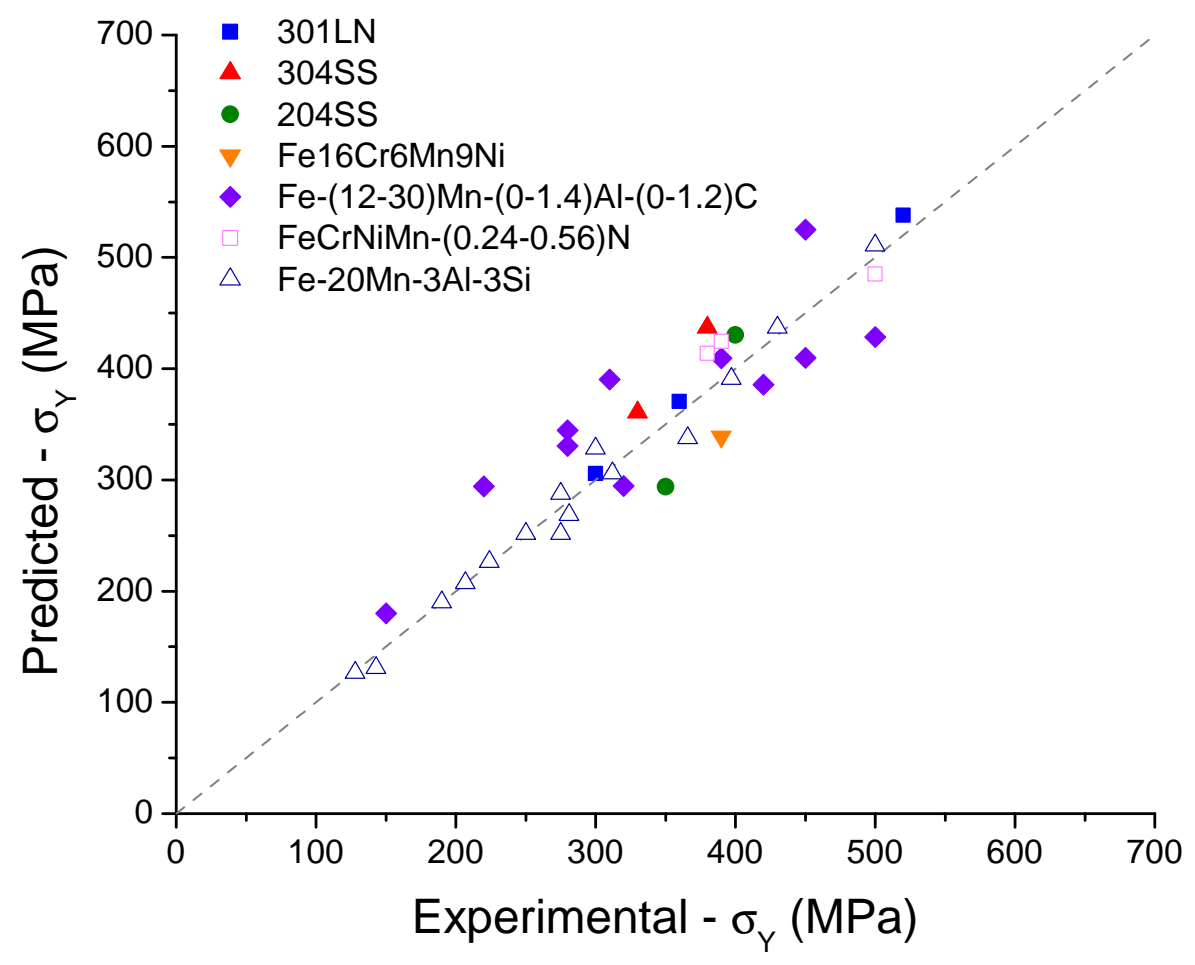

Figure 3: Experimental vs predicted yield stress. 

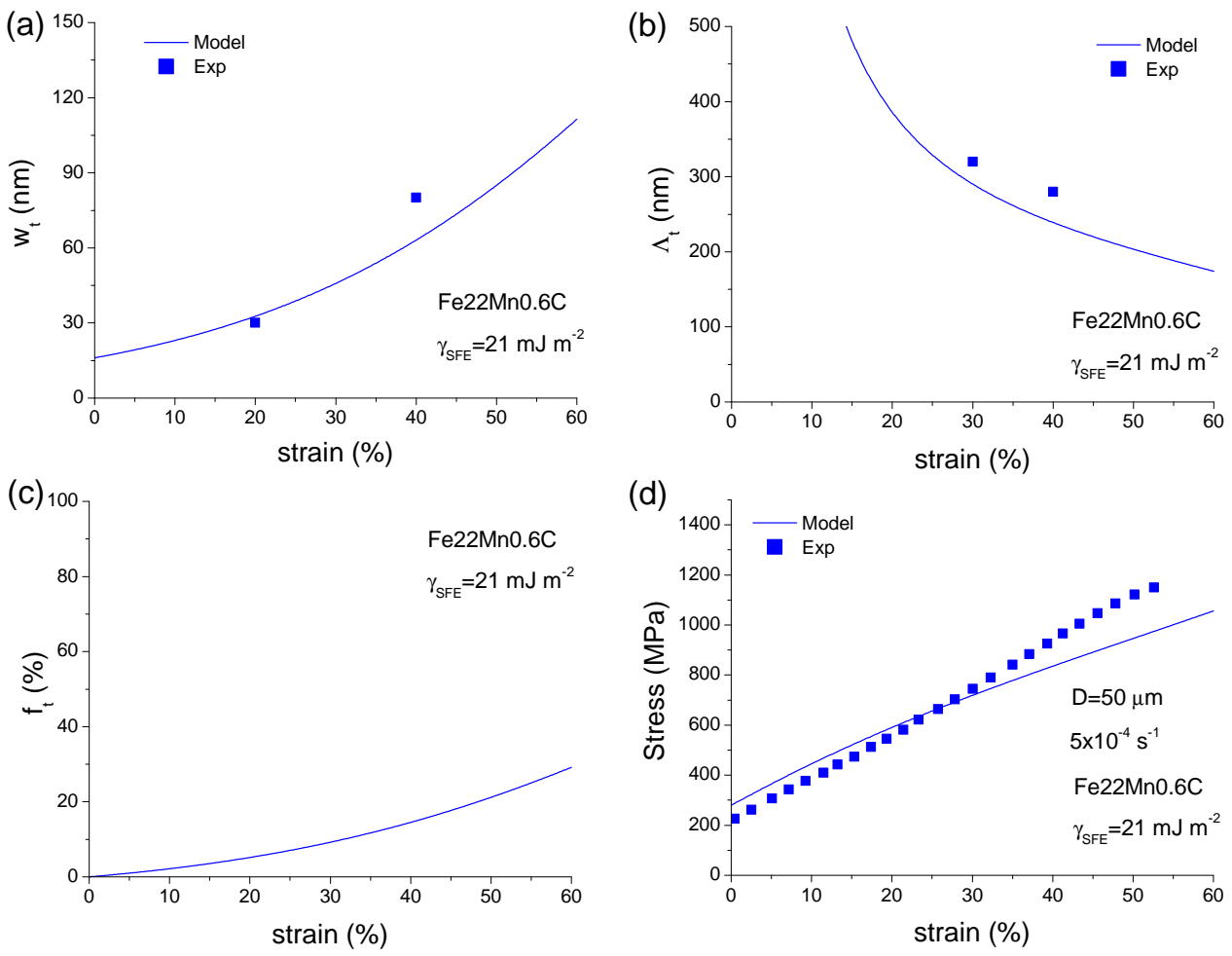

Figure 4: Predicted twinned structure in Fe22Mn0.6C including average twin (a) width, (b) spacing, (c) volume fraction and (d) flow stress response. 

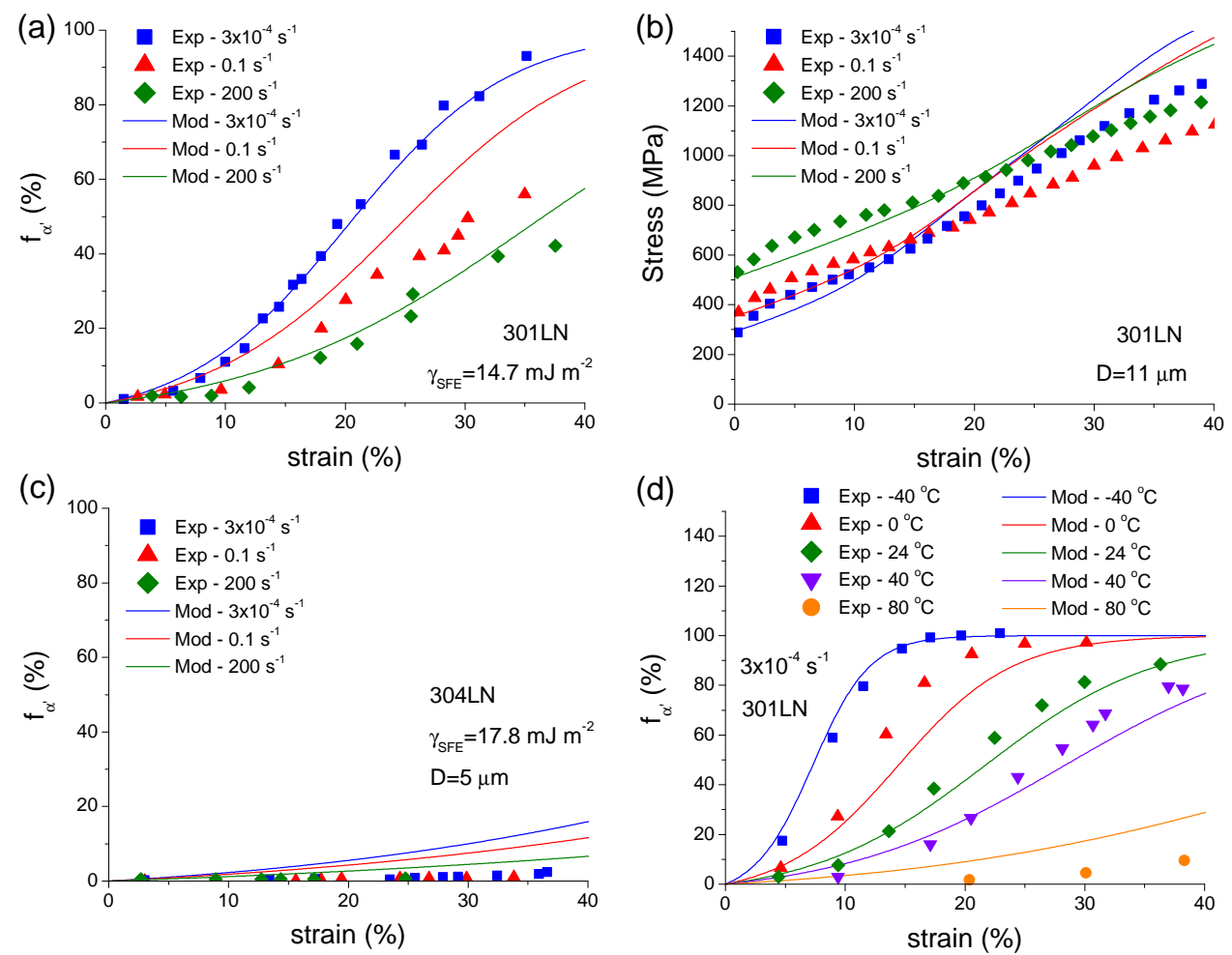

Figure 5: Predicted $\alpha^{\prime}$ evolution in 301LN for different strain rates, including (a) volume fraction and (b) flow stress. (c) $\alpha^{\prime}$ evolution in 304LN at different strain rates. (d) Temperature effects on $f_{\alpha^{\prime}}$ in $301 \mathrm{LN}$. 

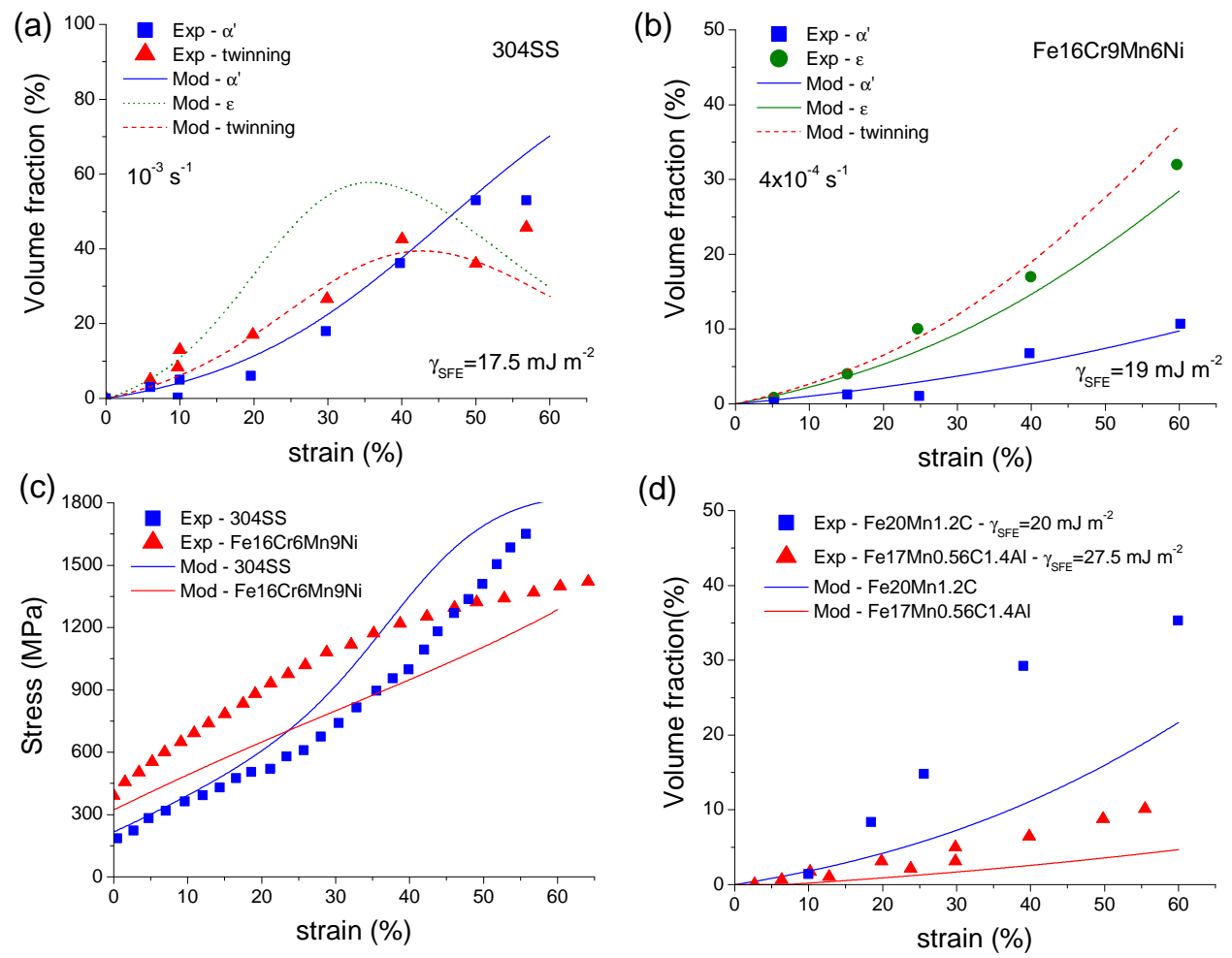

Figure 6: Microstructure evolution in (a) 304SS and (b) Fe16Cr9Mn6Ni1Si when $\epsilon, \alpha^{\prime}$ and twinning operate, and (c) their respective mechanical response. (d) Twin volume fraction in C-containing TWIP steels when only twinning operates. 

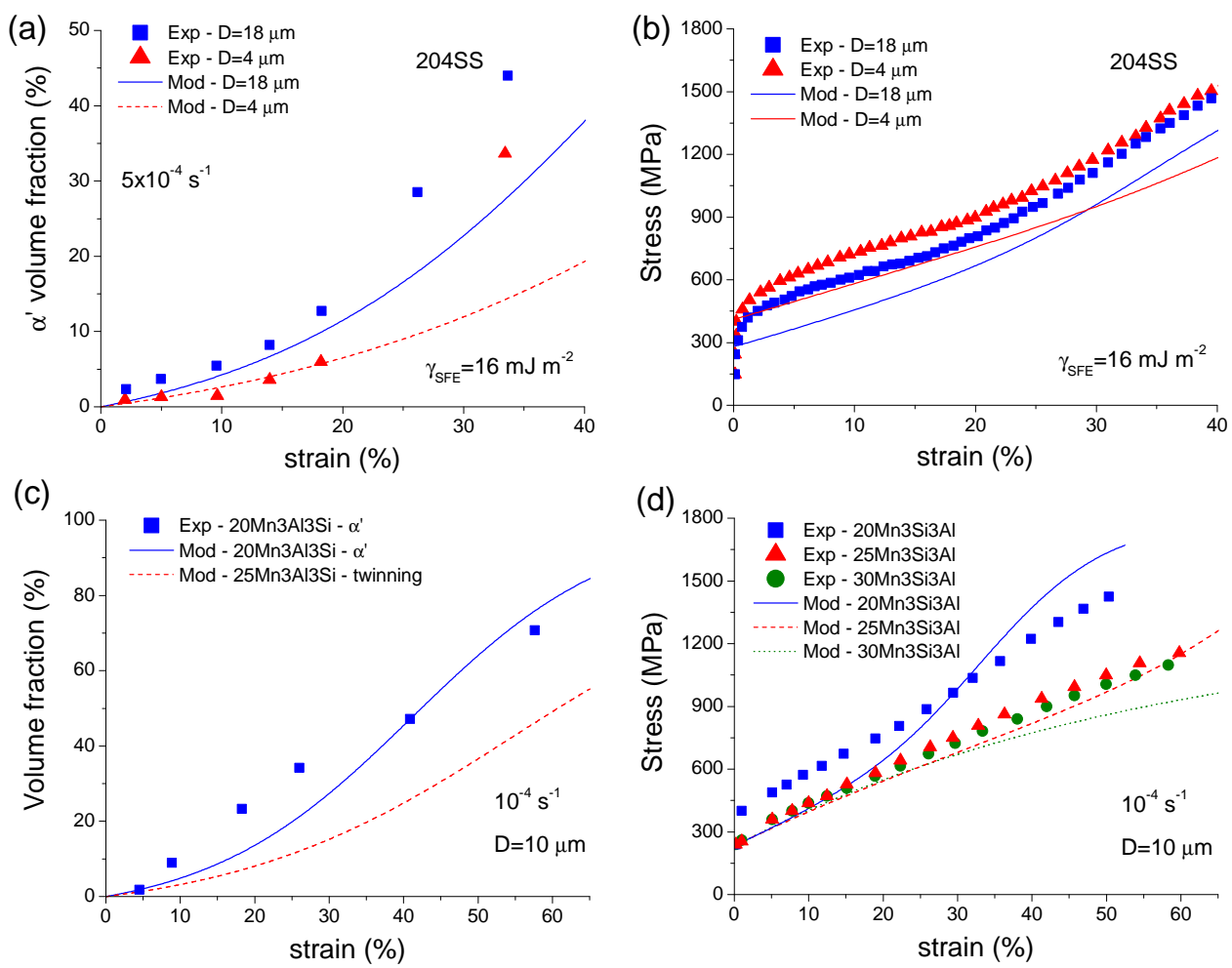

Figure 7: (a) $\alpha^{\prime}$ evolution and (b) flow stress response in 204SS with different grain sizes. (c) Microstructure evolution and the corresponding (d) flow stress evolution in Fe-(20-30)Mn3Si3Al. 

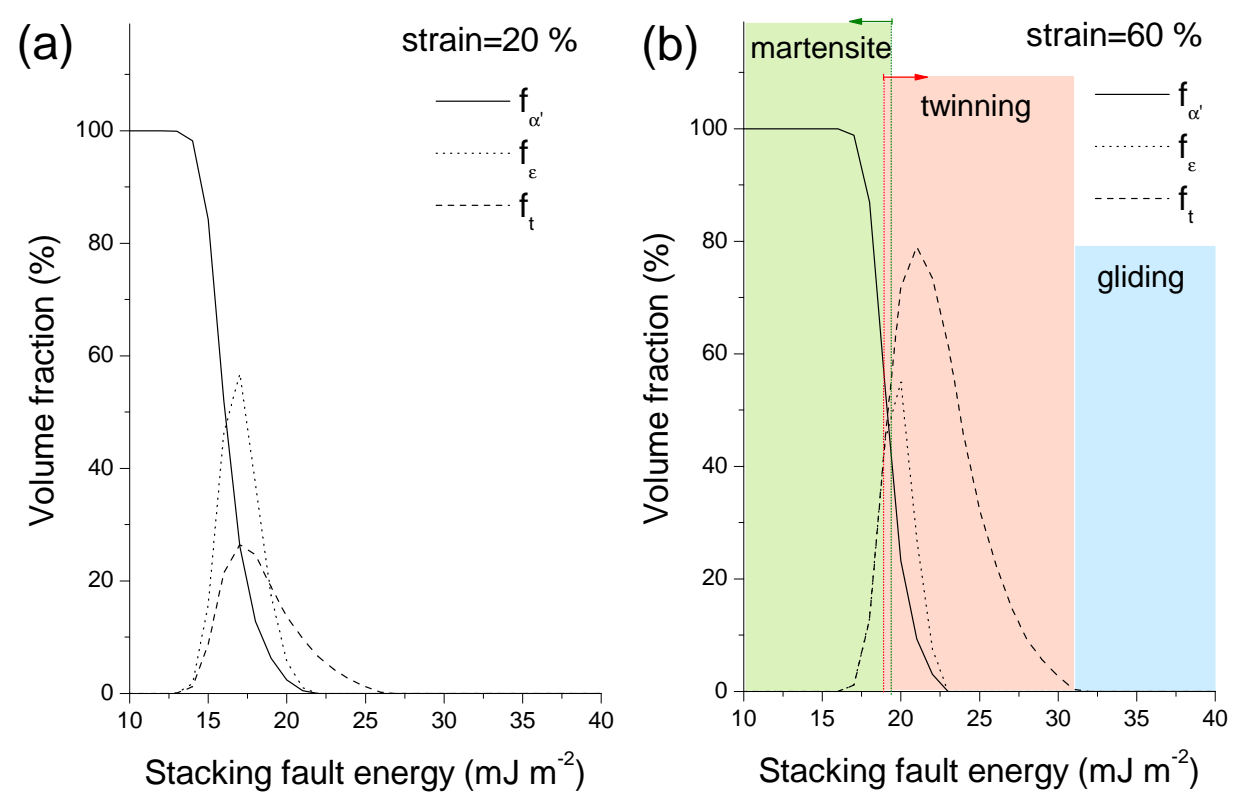

Figure 8: Variation in the volume fraction of $\alpha^{\prime}, \epsilon$ and twinning for different stacking fault energies at (a) $\varepsilon=20 \%$ and (b) $\varepsilon=60 \%$. 

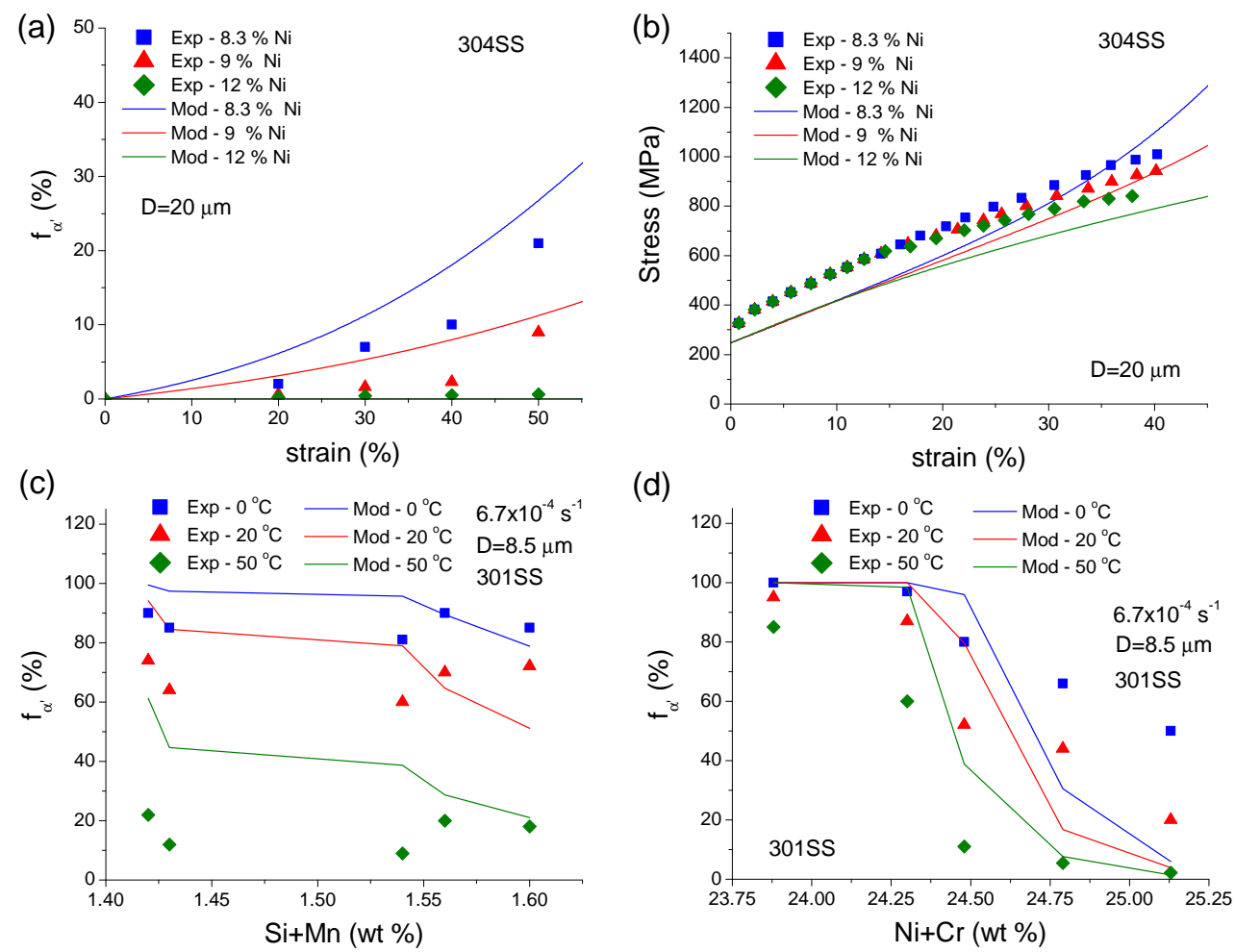

Figure 9: (a) $\alpha^{\prime}$ fraction and (b) flow stress in 304SS with different Ni contents. $\alpha^{\prime}$ fraction in 301SS when modifying simultaneously (c) Si and Mn and (d) Ni and Cr. 